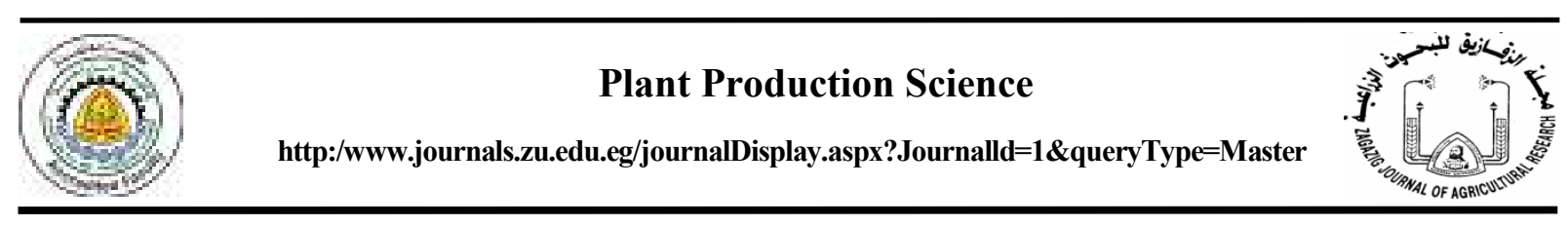

\title{
EFFECT OF SOME AGRONOMIC PRACTICES ON YIELD AND ITS ATTRIBUTES OF SOME YELLOW MAIZE HYBRIDS
}

\author{
Mohamed A.I. Sham", O.A. Zeiton, A.A. El-Khawaga and S.A. Mowafy \\ Agron. Dept., Fac. Agric., Zagazig Univ., Egypt
}

Received: 22/04/2018 ; Accepted: 15/07/2018

\begin{abstract}
Two field experiments were carried out in an administration field at Sheet Al Hawa Village, Kafr Sakr district, Sharkia Governorate, Egypt, during the two successive summer seasons of 2013 and 2014 to find out the effect of four $\mathrm{N}$ levels $(30,60,90$ and $120 \mathrm{~kg} \mathrm{~N} / \mathrm{fad}$.) as well as three phosphorus fertilization regimes $\left(30 \mathrm{~kg} \mathrm{P}_{2} \mathrm{O}_{5}\right.$ fad., phosphorien and $15 \mathrm{~kg} \mathrm{P}_{2} \mathrm{O}_{5} /$ fad., with phosphorien) on yield potentiality of three yellow maize hybrids (SC 168, SC167 and TWC 352). The results could be summered as follows: In case of douple eared plants: Results revealed that the three maize hybrids gave significant differences in the majority of the studied characters of maize crop, since the SC 167 hybrid was superior in most yield attributes as it recorded high value in each of ear length, number of grains/row and hundred grain weight, followed by SC 168 and TWC 352 in descending order. Results of the combined analysis revealed that each $\mathrm{N}$ increment from 30 to 60 and 90 and then to $120 \mathrm{~kg}$ $\mathrm{N} /$ fad., significantly increased ear length, but this response was up to $90 \mathrm{~kg} \mathrm{~N} /$ fad., in each of number of grains/row and number of rows/ear, whereas hundred grain weight responded up to $30 \mathrm{~kg} \mathrm{~N} / \mathrm{fad}$., Addition of $15 \mathrm{~kg} \mathrm{P}_{2} \mathrm{O}_{5} / \mathrm{fad}$., with phosphorien gave high value in each of ear length, number of grains/row, number of rows/ear and hundred grain weight. In case of mono eared plans: The findings revealed that the three maize hybrids gave significant differences in the majority of the studied characters of maize crop, since the SC 167 hybrid was superior in most yield attributes and grain yield/ fad., followed by SC 168 as well as TWC 352. This superiority was expressed in ear length, number of grains/row, hundred grain weight, ear grain weight, while TWC 352 was superior in number of rows/ ear. Results of the combined analysis revealed that each nitrogen increment up to $90 \mathrm{~kg} \mathrm{~N} / \mathrm{fad}$., was accompanied by a significant increase in ear length, number of grains/row, whereas each of number of rows/ear and ear grain weight as well as grain yield/fad., responded up to $120 \mathrm{~kg} \mathrm{~N} /$ fad. In addition, the studied treatments showed that the application of $15 \mathrm{~kg} \mathrm{P}_{2} \mathrm{O}_{5} /$ fad., along with bio- fertilizer phosphorien gave the highest mean records in each of ear length, number of rows/row, hundred grain weight, ear grain weight whereas when maize plants were fertilized by $30 \mathrm{~kg} \mathrm{P}_{2} \mathrm{O}_{5} /$ fad., or phosphorien only gave higher grain yield/fad. The most interaction effect was observed between maize hybrids on one hand and each of $\mathrm{N}$ level and phosphorus treatments on the other hand. SC 167 had the highest grain yield averages under both $120 \mathrm{~kg} \mathrm{~N} /$ fad., and addition of phosphorien, whereas TWC 352 recorded the lowest grain yield under $30 \mathrm{~kg} \mathrm{~N}$ and phosphrien only.
\end{abstract}

Key words: N-level, hybrids, corn, biofertilizer, phosphorien.

\section{INTRODUCTION}

Maize is one of the most important cereal crops under global cultivation and in Egypt. Maize is considered as one of the main cereal crops, ranks the third after wheat and rice. Increasing productivity of land area can be achieved by cultivating high yield cultivars and hybrids paralleled with improved agronomic practices. Several investigators showed that maize hybrids were significantly different in grain yield and its components (Abd ElMaksoud and Sarhan, 2008; Attia et al., 2009; Abdou et al., 2012; Mukhtar et al., 2012;

Corresponding author: Tel. : +201064631584

E-mail address: abdelsatarkhawaga@yahoo.com 
Radma and Dagsh, 2013; Modhej et al., 2014). In this respect, Sharifi et al. (2009) showed that maize hybrids (SC 504, SC 404 and DC 370) significantly differed in plant height, stem diameter, number of kernels row ${ }^{-1}$, cob length, number of kernels ear ${ }^{-1}$ and grain yield fad. ${ }^{-1}$, Golezani and Tajbakhsh (2012) reported that the highest grains per plant and grain yield were obtained by SC- 604, followed by SC-500, OSSK-602 and DC-370, respectively. Maximum grain weight of maize hybrids mainly influenced by grain filling rate rather than by grain filling duration. Kandil (2013) indicated that maize hybrids exhibited significant differences in maize growth, crop yield and its components. Wherein hybrid, SC 10 showed the maximum values of all parameters without significant difference with TWC 329 in most of characteristics over other two maize hybrids (SC 122 and SC 129). Hejazi and Soleymain (2014) indicated that several hybrids of maize (Single cross 704, single cross 640 and single cross 540) were significantly differed in number of rows per ear, number of grains per row, number of grains per ear, grain weight per ear and grain yield. Sorkhi and Fateh (2014) found that number of ears $/ \mathrm{m}^{2}$ of SC 320 hybrid was significantly higher than SC 301 hybrid, but number of grains ear ${ }^{-1}$ and 1000- grain weight in SC 320 hybrid was significantly lower than SC 301 hybrid.

Nitrogen is one of the most important nutrients limiting maize yield in various parts of the world. Accurate fertilizer $\mathrm{N}$ recommendations for yellow maize production for important maximizing productivity. Several researches reported a beneficial effect for $\mathrm{N}$ application to maize among them (Darwich, 2013; El-Moursy, 2013; Kandil, 2013). The results of some research work showed that the optimum $\mathrm{N}$ fertilizer level varied in amounts ranging between 90 and $100 \mathrm{Kg} \mathrm{N} / \mathrm{fad}$. (Hejazi and Soleymani, 2014; Khan et al., 2014; Modhej et al., 2014; Olusegun, 2015; Seadh et al., 2015). Moreover, other studies stressed the need of maize plants to $\mathrm{N}$ fertilizer levels up to $120 \mathrm{Kg}$ N/fad., (El-Sobky, 2014; Sorkhi and Fateh, 2014; El-Kholy, 2015; Ikramullah et al., 2015). In all these responses, the significant increase of yield was attributed to the significant increase of yield attributes.
Phosphorus, also, plays a central role in plants in energy transfer and protein metabolism. Because of the rapid fixation of available $\mathrm{P}$ under Egyptian soil conditions, the application of $\mathrm{P}$ before planting is a common practice. some authors reported significant increase in yield and attributes of maize due to the increase of $\mathrm{P}$ level up to $30 \mathrm{~kg} \mathrm{P}_{2} \mathrm{O}_{5} /$ fad., (Alias et al., 2003; Iqbal and Chauhan, 2003; Khan et al., 2005; Ahmad et al., 2007). Other authors reported that maize responded to more additions of $\mathrm{P}$ reaching $35 \mathrm{Kg} \mathrm{P}_{2} \mathrm{O}_{5} /$ fad. (Hussain et al., 2007; Salih et al., 2007; Yosefi and Mousari, 2011; Zafar et al., 2011).

Bio-fertilizers usually contains microorganisms having specific function such as $\mathrm{P}$ solubilizing bacteria to solubilize $\mathrm{P}$ from the soil and fertilizers to be available to plants. Lin et al. (1983) noticed that increasing yield was attributed to the plant growth promoting substances by root colonizing bacteria more than the biological nitrogen fixation, stated that yield increased due to promoting root growth which in turn enchancing nutrients and water uptake from the soil. Glass (1989) noticed that, The low availability of $P$ to plants is because the vast majority of soil P is found in insoluble forms, and plants can only absorb $\mathrm{P}$ in two soluble forms, the monobasic $\left(\mathrm{H}_{2} \mathrm{PO}_{4}\right)$ and the dibasic $\left(\mathrm{HPO}_{4}\right)^{2-}$ ions. Chabot et al. (1993) recorded that, application of biofertilizers increased growth and yield in many researches. Karimian (2000) noticed that: Grain crops in general, and corn in particular have high demand for chemical fertilizers. Therefore, application of biological products to supply grain with nutrients is essential for a good yield and to improve quality of agricultural produce, production stability and thus making a contribution to national food security in the society at large without the release of chemical toxins. Jat and Shaktawat (2003) confirmed satisfactory results from the use of phosphate biological fertilizer, compared to triple super phosphate fertilizer in corn, soybean and wheat crops, confirming the satisfactory effect of this fertilizer. It was also clear that phosphate biological fertilizer leads to a considerable increase in yield. Therefore, based on existing experience and evidence, it is crucial that 
phosphate fertilizer is supplemented with microorganisms that can solve phosphate and maximize the effect of phosphorus absorption for decreasing chemical fertilizer consumption (Behbahani and Khayyam, 2004). Bolan and Duraisamy (2003) described that application of phosphate solubilizing bacteria (PSB) play a significant part in phosphorus nutrition by improving phosphorus accessibility to the plants through the release of organic and inorganic soil phosphorus pools by mineralization and solubilizing bacteria as inoculants enchance phosphorus uptake. Pradhan and Sukla (2005) recorded that, the organisms possessing phosphate solubilizing ability called phosphate solubilizing organisms (PSMs), which are reterred to a group to soil microorganisms that are a component of $\mathrm{P}$ cycle, capable of dissolving insoluble forms of phosphates into plant available forms. Several groups of microorganisms including fungi, bacteria and actinomycetes are known as efficient fixed P solubilizers, Sundara et al. (2002).

Shevananda (2008) recorded that, application of biofertilizers became of great necessity to get a yield of high quality and to avoid the environmental pollution. Xiao et al. (2011) observed that phosphorus, is the second most important macronutrient required by the plants, next to nitrogen, is reported to be a critical factor of many crop production systems, due to the fact that the limited availability in soluble forms in the soils. Beyranvand et al. (2013) suggested that effect of nitrogen and phosphate biofertilizers were evaluated positively, there were an increase in plant height, ear weight, and number of grains per cob, grain yield and biomass yield.

\section{MATERIALS AND METHODS}

Two field experiments were carried out in an administration field at Sheet Al Hawa Village, Kafr Saker District, Sharkia Governorate, Egypt, during the two successive summer seasons of 2013 and 2014 to find out the response of three maize hybrids (Zea mays L.) to different levels of nitrogen fertilizer and addition of phosphorus fertilizer and the biofertilizer phosphorien.

\section{Studied Factors}

Maize hybrids (H), (Main plots)

1- Yellow single cross ( SC) 168

2- Yellow single cross ( SC) 167

3- Yellow three way cross (TWC) 352

The three maize hybrids were kindly released by maize Res. Dept., ARC.

\section{Nitrogen levels}

The four nitrogen levels were 30,60, 90 and $120 \mathrm{~kg} \mathrm{~N} /$ fad.

\section{Phosphorus fertilization regimes (Sub-sub plots)}

1- $30 \mathrm{Kg} \mathrm{P}_{2} \mathrm{O}_{5} /$ fad. 2- Biofertilizer phosphorien

3- $15 \mathrm{~kg} \mathrm{P}_{2} \mathrm{O}_{5} /$ fad., + phosphorien

Each plot consisted of six ridges, each ridge was $3 \mathrm{~m}$ long, $80 \mathrm{~cm}$ in width $25 \mathrm{~cm}$ between hills and one blank ridge was left between plots. The outer two ridges $\left(1^{\text {st }}\right.$ and $6^{\text {th }}$ ) were considered as borders. The previous crop was faba bean in both years. Planting was done on June $4^{\text {th }}$ in 2013 season, and June $3^{\text {rd }}$ in 2014 season. Phosphorus was applied in form of ordinary super phosphate $\left(\begin{array}{lll}15.5 \% & \mathrm{P}_{2} \mathrm{O}_{5}\end{array}\right)$ and applied at the mentioned levels before planting, phosphorien wass mixed with seeds just before sowing. Two grains were hand planted in each hill. Thinning to one plant per hill was done before the first irrigation. Hoeing twice was done for controlling weeds before the first and second irrigations, respectively.

Nitrogen fertilizer in form of urea $(46.6 \% \mathrm{~N})$, was applied in two equal doses according to experimental rates before the first and the second irrigations, respectively. Recommended pest control was applied when necessary.

\section{Experimental Design}

A split-split plot design of three replications was used. Main plots were occupied by the three maize hybrids, nitrogen fertilizer levels in subplots and phosphorus fertilization regimes were in sub sub plots according to Steel and Torrie (1980).

\section{Soil Sampling and Analysis}

The soil samples of the experimental fields in both seasons were laboratory analyzed and their physical and chemical properties are shown in 
Table 1. Both physical and chemical analyses of the soil used were carried out by following the method described by Jackson (1958). Whereas $\mathrm{N}, \mathrm{P}$ and $\mathrm{K}$ elements as well as some micronutrients were determined by applying the procedure documented by Lindsay and Norvell (1978).

\section{Studied Characters}

\section{Yield and yield attributes}

At harvest, the following yield attributs were recorded on ten plants and ears:

1- Ear length when maize plants carried one or douple eared plants.

2- Ear diameter: It was measured by using a varnier caliper as the means of random five ears.

3- Number of rows/ear when maize plants carried one or douple eared plants.

4- Number of grains/row when maize plants carried one or two ears/plant.
5- Ear grain weight.

6- Hundred grain weight for plants which carried one or two ears/plant.

7- Grain yield in ardab per faddan. It was recorded at harvest from the second and thrid ridges of each plot. Grain yield was adjusted to moisture content of $15-5 \%$ and transformed to ardab per faddan (one ardab $=140 \mathrm{~kg}$ and one faddan $4200 \mathrm{~m}^{2}$ ).

\section{Statistical Analysis}

The date were statistically analyzed according to Gomez and Gomez (1984), using computer MSTAT- C statistical analysis package (Freed et al., 1989). Treatment meani were compared according to the LSD test. In the tables of the analysis of variance $*, * *$ indicate significance at 0.05 and 0.01 levels of probability, respectively as described by Waller and Duncan's (1969).

Table 1. The physical and some chemical properties of the experimental site $(0-30 \mathrm{~cm}$ depth) averages of both seasons

\begin{tabular}{lcc}
\hline Property & $\mathbf{2 0 1 3}$ & $\mathbf{2 0 1 4}$ \\
\hline Mechanical analysis & 24.55 & 24.80 \\
Sand (\%) & 27.80 & 28.70 \\
Silt (\%) & 47.55 & 46.50 \\
Clay (\%) & Clay & Clay \\
Soil texture & & \\
Chemical analysis & 8.20 & 8.75 \\
pH & 1.75 & 1.45 \\
OM (\%) & 28.0 & 35.0 \\
Available N (ppm) & 9.11 & 8.12 \\
Available P (ppm) & 1150 & 1100 \\
Avaidable K (ppm) & 2.25 & 2.15 \\
Ecmm/cm & & \\
\hline
\end{tabular}

Central Laboratory of Faculty of Agriculture, Zagazig University, Egypt. 


\section{RESULTS AND DISCUSSION}

\section{Yield and Yield Attributes}

\section{Ear length (cm)}

Results presented in Table 2 reflect the influence of $\mathrm{N}$ fertilizer levels and addition of $\mathrm{P}$ levels and phosphorien on ear length of three yellow maize hybrids when maize plants carried one or douple eared plants.

\section{Maize hybrids differences}

In both seasons and their combined, SC 168 and SC 167 had higher ear length value than TWC 352 in mono eared plants. But in douple eared plants, the results indicated clearly that SC 167 was always superior in ear length. This was completely true in the first season and their comained analysis. The differences in ear length among the three maize hybrids might be attributed to the genetically variation. Similar maize hybrids changes in ear length was obtained by Abd El-Maksoud and Sarhan (2008), Atiaa et al. (2009), Abdou et al. (2012) and Mukhtar et al. (2012).

\section{Nitrogen levels effect}

It is evident from Table 2 that each $\mathrm{N}$ increment up to $120 \mathrm{~kg} \mathrm{~N} /$ fad., produced a significant increase in ear length of the douple eared plants in both seasons and their combined, but application of $90 \mathrm{~kg} \mathrm{~N} /$ fad., was quite enough to produce the highest average ear length when maize plants carried one ear/plant in the second season and their combined. Superiority in ear length with application of $\mathrm{N}$ fertilizer could be ascribed to the role of nitrogen in stimulating the capacity of plants in building metabolites and this might account much for the superiority in ear length.

Also, it is worth to note that nitrogen effect on ear length could be expounded by its role in encouraging amino acids synthesis including tryptophan, which considered the major substrate for indol acetic acid (IAA) which help ears to grow longer.

Increasing ear length of maize due to $\mathrm{N}$ fertilizer application was also reported by El-Moursy (2013), Kandil (2013), Khan et al. (2014) and Hejazi and Soleymain (2014).

\section{Phosphorus fertilizer and phosphorien}

Significant differences in maize ear length due to phosphorus fertilizer and addition of phosphorien were observed in the two seasons and their combined when maize plants carried one or two ears/plant. Treated plants with $15 \mathrm{~kg}$ $\mathrm{P}_{2} \mathrm{O}_{5}+$ phosphorien ranked first in ear length when carried two ears/plant while addition of $30 \mathrm{~kg} \mathrm{P}_{2} \mathrm{O}_{5} / \mathrm{fad}$., came in the second rank, but addition of phosphorien only ranked third without significant differences between phosphorien and addition of $15 \mathrm{~kg} \mathrm{P}_{2} \mathrm{O}_{5}+$ phosphorien in mono eared plants in the two seasons.

The increasing of ear length with chemical $P$ fertilizer was also reported by Alias et al. (2003), Iqbal and Chauhan (2003) and Khan et al. (2005).

The positive impact of phosphorus chemical fertilization on maize ear length was also confirmed by Lin et al. (1983) and Glass (1989).

\section{Interaction effect}

It is evident from Table 2-a that growing maize under both 60 and $120 \mathrm{~kg} \mathrm{~N} /$ fad., ear length of SC 167 was longer than SC 168 and TWC 352 in both mono and douple eared plants. In this respect, it is noticed that ear length of SC 167 responded to $\mathrm{N}$ fertilizer more than each of SC 168 and TWC 352 when maize plants carried one ear/plant, but when carried two ears/ plant the three maize hybrids responded to $\mathrm{N}$ fertilizer up to $120 \mathrm{~kg} \mathrm{~N} / \mathrm{fad}$.

SC 168 hybrid had longer ears compared with SC 167 and TWC 352 under each of $30 \mathrm{k}$ $\mathrm{P}_{2} \mathrm{O}_{5} /$ fad., and phosphorien whereas under $15 \mathrm{~kg}$ $\mathrm{P}_{2} \mathrm{O}_{5} /$ fad., + phosphorien SC 167 had longer ear length than the other two hybrids in one ear/ plant.

When maize plants carried two ears/ plant the three maize hybrids had longer ear length under $15 \mathrm{~kg} \mathrm{P}_{2} \mathrm{O}_{5} /$ fad., + phosphorien, on other hand, SC 167 had longer ear length under phosphorien only or $15 \quad \mathrm{~kg} \quad \mathrm{P}_{2} \mathrm{O}_{5} /$ fad., + phosphorien. Maize hybrids SC 168 and SC 167 had the same ear length when maize was grown under $30 \mathrm{~kg} \mathrm{P}_{2} \mathrm{O}_{5}$ (Table 2-b). 
Table 2. Ear length (cm) of maize as influenced by the different treatments during 2013 and 2014 seasons

\begin{tabular}{|c|c|c|c|c|c|c|}
\hline \multirow[t]{2}{*}{ Main effects and interactions } & \multicolumn{3}{|c|}{$\begin{array}{c}\text { Ear length }(\mathrm{cm}) \\
\text { mono eared plants }\end{array}$} & \multicolumn{3}{|c|}{$\begin{array}{c}\text { Ear length (cm) } \\
\text { douple eared plants }\end{array}$} \\
\hline & 2013 & 2014 & Combined & 2013 & 2014 & Combined \\
\hline \multicolumn{7}{|l|}{ Maize hybrids (H) } \\
\hline SC 168 & $24.18 \mathrm{a}$ & $24.72 \mathrm{a}$ & $24.45 \mathrm{a}$ & $22.43 b$ & $22.30 \mathrm{a}$ & $22.37 b$ \\
\hline SC 167 & $24.08 \mathrm{a}$ & $24.68 \mathrm{a}$ & $24.38 \mathrm{a}$ & $22.95 \mathrm{a}$ & $22.33 a$ & $22.64 a$ \\
\hline TWC 352 & $19.76 b$ & $20.30 \mathrm{~b}$ & $20.02 b$ & $18.74 \mathrm{c}$ & $18.64 b$ & $18.69 \mathrm{c}$ \\
\hline F. test & $* *$ & $* *$ & $* *$ & $* *$ & $* *$ & $* *$ \\
\hline \multicolumn{7}{|l|}{ Nitrogen level (kg N/fad.) (N) } \\
\hline 30 & $22.41 b$ & $22.50 \mathrm{c}$ & $22.45 d$ & $21.17 \mathrm{c}$ & $20.25 \mathrm{~d}$ & $20.71 d$ \\
\hline 60 & $22.97 \mathrm{a}$ & $22.75 b$ & $22.86 \mathrm{c}$ & $20.96 \mathrm{~d}$ & $20.83 \mathrm{c}$ & $20.89 \mathrm{c}$ \\
\hline 90 & $22.86 a$ & $23.82 \mathrm{a}$ & $23.34 \mathrm{a}$ & $21.45 b$ & $21.33 b$ & $21.39 b$ \\
\hline 120 & $22.46 \mathrm{a}$ & $23.86 \mathrm{a}$ & $23.16 b$ & $21.91 \mathrm{a}$ & $21.95 \mathrm{a}$ & $21.93 \mathrm{a}$ \\
\hline F. test & $* *$ & $* *$ & $* *$ & $* *$ & $* *$ & $* *$ \\
\hline \multicolumn{7}{|l|}{ Phosphorus fertilization regimes (p) } \\
\hline $30 \mathrm{~kg} \mathrm{P}_{2} \mathrm{O}_{5} /$ fad & $22.53 b$ & $22.73 b$ & $22.63 \mathrm{c}$ & $21.32 b$ & $21.03 b$ & $21.21 b$ \\
\hline Phosphorien & $22.70 \mathrm{a}$ & $23.44 a$ & $23.07 \mathrm{~b}$ & $20.91 \mathrm{c}$ & $20.44 \mathrm{c}$ & $20.67 \mathrm{c}$ \\
\hline $15 \mathrm{~kg} \mathrm{P}_{2} \mathrm{O}_{5} /$ fad. + phosphorien & $22.80 \mathrm{a}$ & $23.53 \mathrm{a}$ & $23.16 \mathrm{a}$ & $21.90 \mathrm{a}$ & $21.74 \mathrm{a}$ & $21.82 \mathrm{a}$ \\
\hline F. test & $* *$ & $* *$ & $* *$ & $* *$ & $* *$ & $* *$ \\
\hline \multicolumn{7}{|l|}{ Interactions } \\
\hline $\mathbf{H} \times \mathbf{N}$ & $* *$ & $* *$ & $* *$ & $* *$ & $* *$ & $* *$ \\
\hline $\mathbf{H} \times \mathbf{P}$ & $* *$ & $* *$ & $* *$ & $* *$ & $* *$ & $* *$ \\
\hline $\mathbf{N} \times \mathbf{P}$ & $* *$ & $* *$ & $* *$ & $* *$ & $* *$ & $* *$ \\
\hline
\end{tabular}

Table 2-a. Effect of interaction between $\mathbf{N}$ fertilizer levels and maize hybrids on ear length (combined data)

\begin{tabular}{lcccc}
\hline Maize hybrids (H) & \multicolumn{4}{c}{ N levels (kg N/fad.) } \\
\cline { 2 - 5 } & $\mathbf{3 0}$ & $\mathbf{6 0}$ & $\mathbf{9 0}$ & $\mathbf{1 2 0}$ \\
\hline & $\mathrm{C}$ & \multicolumn{2}{c}{ Mono eared plants } & \\
SC 168 & $23.74 \mathrm{a}$ & $24.32 \mathrm{~b}$ & $25.31 \mathrm{a}$ & $24.43 \mathrm{~b}$ \\
& $\mathrm{D}$ & $\mathrm{B}$ & $\mathrm{C}$ & $\mathrm{A}$ \\
SC 167 & $23.59 \mathrm{a}$ & $24.55 \mathrm{a}$ & $24.26 \mathrm{~b}$ & $25.11 \mathrm{a}$ \\
& $\mathrm{B}$ & $\mathrm{C}$ & $\mathrm{A}$ & $\mathrm{BC}$ \\
TWC 352 & $20.04 \mathrm{~b}$ & $19.7 \mathrm{c}$ & $20.44 \mathrm{c}$ & $19.44 \mathrm{c}$ \\
& & Douple eared & plants & \\
SC 168 & $\mathrm{D}$ & $\mathrm{B}$ & $\mathrm{C}$ & $\mathrm{A}$ \\
& $21.30 \mathrm{~b}$ & $22.50 \mathrm{~b}$ & $22.37 \mathrm{~b}$ & $23.29 \mathrm{a}$ \\
SC 167 & $\mathrm{C}$ & $\mathrm{B}$ & $\mathrm{B}$ & $\mathrm{A}$ \\
& $21.67 \mathrm{a}$ & $22.81 \mathrm{a}$ & $22.86 \mathrm{a}$ & $23.21 \mathrm{a}$ \\
TWC 352 & $\mathrm{B}$ & $\mathrm{D}$ & $\mathrm{C}$ & $\mathrm{A}$ \\
& $19.16 \mathrm{c}$ & $17.37 \mathrm{c}$ & $18.95 \mathrm{c}$ & $19.29 \mathrm{~b}$ \\
\hline
\end{tabular}


Table 2-b. Effect of interaction between maize hybrids and phosphorus fertilization regimes on ear length (combined data)

\begin{tabular}{|c|c|c|c|}
\hline \multirow[t]{2}{*}{ Maize hybrid (H) } & \multicolumn{3}{|c|}{ Phosphorus fertilization regimes } \\
\hline & $30 \mathrm{~kg} \mathrm{P}_{2} \mathrm{O}_{5} /$ fad. & Phosphorien & $15 \mathrm{~kg} \mathrm{P}_{2} \mathrm{O}_{5} /$ fad. + phosphorien \\
\hline & \multicolumn{3}{|c|}{ Mono eared plants } \\
\hline & B & A & A \\
\hline \multirow[t]{2}{*}{ SC 168} & $24.33 \mathrm{a}$ & $24.56 \mathrm{a}$ & $24.46 b$ \\
\hline & $\mathrm{C}$ & B & A \\
\hline \multirow[t]{2}{*}{ SC 167} & $24.14 b$ & $24.33 b$ & $24.67 \mathrm{a}$ \\
\hline & $\mathrm{B}$ & A & A \\
\hline \multirow[t]{3}{*}{ TWC 352} & $19.42 \mathrm{c}$ & $20.3 \mathrm{c}$ & $20.36 c$ \\
\hline & \multicolumn{3}{|c|}{ Douple eared plants } \\
\hline & B & $\mathrm{C}$ & A \\
\hline \multirow[t]{2}{*}{ SC 168} & $22.59 \mathrm{a}$ & $21.45 b$ & $23.06 \mathrm{~b}$ \\
\hline & $\mathrm{B}$ & $\mathrm{C}$ & A \\
\hline \multirow[t]{2}{*}{ SC 167} & $22.62 \mathrm{a}$ & $22.11 \mathrm{a}$ & $23.19 \mathrm{a}$ \\
\hline & $\mathrm{B}$ & B & A \\
\hline TWC 352 & $18.72 b$ & $18.46 \mathrm{c}$ & $19.20 \mathrm{c}$ \\
\hline
\end{tabular}

It is evident from Table 2-c, that the application of $90 \mathrm{~kg} \mathrm{~N} /$ fad., under any phosphorus fertilizer used, produced the longest ear length when maize plant carried one ear/plant, moreover, ears became longer under $120 \mathrm{~kg} \mathrm{~N} /$ fad., in douple eared plants. The uppermost ear length $(23.87$ $\mathrm{cm})$ was obtained under the application of both $90 \mathrm{~kg} \mathrm{~N} /$ fad., and $15 \mathrm{~kg} \mathrm{P}_{2} \mathrm{O}_{5}+$ phosphorien, while the shortest ear length $(22.29 \mathrm{~cm})$ was attained due to application both $30 \mathrm{~kg} \mathrm{~N} / \mathrm{fad}$., and $30 \mathrm{~kg} \mathrm{P}_{2} \mathrm{O}_{5} /$ fad., in mono eared plants. But increasing $\mathrm{N}$ level up to $120 \mathrm{~kg} \mathrm{~N} / \mathrm{fad}$., tended to produce longer ears with different $\mathrm{p}$ application therefore, the longest ear length was obtained by $15 \mathrm{~kg} \mathrm{P}_{2} \mathrm{O}_{5}+$ phosphorien when $120 \mathrm{~kg} \mathrm{~N} /$ fad., was applied in two ears/plant.

\section{Number of grains/ row}

Results presented in Table 3 show the influence of $\mathrm{N}$ fertilizer levels and addition of $\mathrm{P}$ levels and phosphorien on number of grains/ row of three yellow maize hybrids when maize plants carried one or two ears/plant.

\section{Maize hybrid differences}

Maize hybrids varried significantly in number of grains/row when maize plants carried one or two ears/ plant in both seasons and their combined, while SC 167 and SC 168 had higher number of grains/ row and TWC 352 gave lower values for this character in the two seasons and their combined when maize plant carried one ear/plant. Concerning the number of grains/row when maize plants carried two ears/plant, SC 167 produced higher grain number followed by SC 168, whereas TWC 352 gave the lowest grain number in the two seasons and their combined. These results are in line with those reported by Sharifi et al. (2009), Golezani and Tiajbakhsh (2012), Radma and Dagash (2013), Kandil (2013) and Modhej et al. (2014).

\section{Nitrogen levels effect}

Significant differences in number of grains/ row due to nitrogen fertilization treatments were observed in both seasons and their combined analysis when maize plants carried one or two ears/plant, in the two cases $90 \mathrm{~kg} \mathrm{~N} / \mathrm{fad}$., was a quite enough to produce the largest average of number of grains/row (Table 3 ). 
Table 2-c. Effect of interaction between $\mathbf{N}$ fertilizer levels and phosphorus fertilization regimes on ear length (combined data)

\begin{tabular}{|c|c|c|c|c|}
\hline \multirow[t]{2}{*}{ Phosphorus fertilization regimes } & \multicolumn{4}{|c|}{ N levels (kg N/fad.) } \\
\hline & 30 & 60 & 90 & 120 \\
\hline & \multicolumn{4}{|c|}{ Mono eared plants } \\
\hline & $\mathbf{C}$ & B & $\mathbf{A}$ & $\mathbf{A}$ \\
\hline \multirow[t]{2}{*}{$30 \mathrm{~kg} \mathrm{P}_{2} \mathrm{O}_{5} /$ fad. } & $22.29 b$ & $22.57 b$ & $22.83 \mathrm{c}$ & $22.82 \mathrm{c}$ \\
\hline & $\mathrm{C}$ & A & A & B \\
\hline \multirow[t]{2}{*}{ Phosphorien } & $22.54 \mathrm{a}$ & $23.32 \mathrm{a}$ & $23.31 b$ & $23.10 b$ \\
\hline & $\mathrm{C}$ & $\mathrm{C}$ & A & $\mathrm{B}$ \\
\hline \multirow{3}{*}{$15 \mathrm{~kg} \mathrm{P}_{2} \mathrm{O}_{5} /$ fad. + phosphorien } & $22.53 \mathrm{a}$ & $22.68 b$ & $23.87 \mathrm{a}$ & $23.57 \mathrm{a}$ \\
\hline & \multicolumn{4}{|c|}{ Douple eared plants } \\
\hline & $\mathrm{D}$ & $\mathrm{C}$ & B & A \\
\hline \multirow[t]{2}{*}{$30 \mathrm{~kg} \mathrm{P}_{2} \mathrm{O}_{5} / \mathrm{fad}$} & $20.23 \mathrm{c}$ & $21.09 b$ & $21.71 b$ & $21.80 \mathrm{~b}$ \\
\hline & B & $\mathrm{D}$ & $\mathrm{C}$ & A \\
\hline \multirow[t]{2}{*}{ Phosphorien } & $20.64 b$ & $19.78 \mathrm{c}$ & $20.46 \mathrm{c}$ & $21.82 b$ \\
\hline & $\mathrm{D}$ & $\mathrm{C}$ & $\mathrm{C}$ & A \\
\hline $15 \mathrm{~kg} \mathrm{P}_{2} \mathrm{O}_{5} /$ fad. + phosphorien & $21.27 \mathrm{a}$ & $21.81 \mathrm{a}$ & $22.02 \mathrm{a}$ & $22.17 \mathrm{a}$ \\
\hline
\end{tabular}

Table 3. Number of grains/ row of maize as influenced by the different treatments during 2013 and 2014 seasons

\begin{tabular}{|c|c|c|c|c|c|c|}
\hline \multirow[t]{2}{*}{ Main effects and interactions } & \multicolumn{3}{|c|}{ Mono eared plants } & \multicolumn{3}{|c|}{ Douple eared plants } \\
\hline & 2013 & 2014 & Combined & 2013 & 2014 & Combined \\
\hline \multicolumn{7}{|l|}{ Maize hybrids (H) } \\
\hline SC 168 & $48.64 \mathrm{a}$ & $48.39 \mathrm{a}$ & $48.51 \mathrm{a}$ & $44.33 b$ & $45.33 b$ & $44.83 b$ \\
\hline SC 167 & $48.83 \mathrm{a}$ & $48.47 \mathrm{a}$ & $48.65 \mathrm{a}$ & $45.06 \mathrm{a}$ & $46.28 \mathrm{a}$ & $45.67 \mathrm{a}$ \\
\hline TWC 352 & $39.72 b$ & $39.47 b$ & $39.60 \mathrm{~b}$ & $37.75 \mathrm{c}$ & $39.69 \mathrm{c}$ & $38.72 \mathrm{c}$ \\
\hline F. test & $* *$ & $* *$ & $* *$ & $* *$ & $* *$ & $* *$ \\
\hline \multicolumn{7}{|l|}{ Nitrogen level (kg N/fad.) (N) } \\
\hline 30 & $45.33 c$ & $44.54 b$ & $44.96 \mathrm{c}$ & $42.33 b$ & $43.78 b$ & $43.06 \mathrm{~b}$ \\
\hline 60 & $44.96 \mathrm{~d}$ & $45.0 \mathrm{ab}$ & $44.98 \mathrm{c}$ & $41.52 \mathrm{c}$ & $42.63 b$ & $42.08 \mathrm{c}$ \\
\hline 90 & $46.89 \mathrm{a}$ & $46.52 \mathrm{ab}$ & $46.70 \mathrm{a}$ & $43.59 \mathrm{a}$ & $45.19 \mathrm{a}$ & $44.39 \mathrm{a}$ \\
\hline 120 & $45.74 b$ & $45.67 \mathrm{a}$ & $45.70 \mathrm{~b}$ & $40.07 \mathrm{~b}$ & $43.48 b$ & $42.78 b$ \\
\hline F. test & $* *$ & $* *$ & $* *$ & $* *$ & $* *$ & $* *$ \\
\hline \multicolumn{7}{|l|}{ Phosphorus fertilization regimes (p) } \\
\hline $30 \mathrm{~kg} \mathrm{P}_{2} \mathrm{O}_{5} /$ fad. & $45.53 b$ & $44.47 \mathrm{c}$ & $45.25 \mathrm{c}$ & $41.97 \mathrm{~b}$ & $42.11 b$ & $42.04 \mathrm{c}$ \\
\hline Phosphorien & $46.11 \mathrm{a}$ & $45.86 a$ & $45.99 \mathrm{a}$ & $41.92 b$ & $44.39 \mathrm{a}$ & $43.15 b$ \\
\hline 15kg $\mathrm{P}_{2} \mathrm{O}_{5} /$ fad. + phosphorien & $45.56 \mathrm{~b}$ & $45.5 b$ & $45.53 b$ & $43.25 \mathrm{a}$ & $44.81 \mathrm{a}$ & $44.03 \mathrm{a}$ \\
\hline F. test & $* *$ & $* *$ & $* *$ & $* *$ & $* *$ & $* *$ \\
\hline \multicolumn{7}{|l|}{ Interactions } \\
\hline $\mathbf{H} \times \mathbf{N}$ & $* *$ & & $* *$ & $* *$ & $* *$ & $* *$ \\
\hline $\mathbf{H} \times \mathbf{P}$ & $* *$ & & $* *$ & $* *$ & $* *$ & $* *$ \\
\hline $\mathbf{N} \times \mathbf{P}$ & $* *$ & & ** & ** & ** & ** \\
\hline
\end{tabular}


In the literature, several authors found that addition of nitrogen was effective to increase number of grains/row (El-Sobky, 2014; Sorkhi and Fateh, 2014; El-Kholy, 2015; Ikramullah et al., 2015).

\section{Phosphorus fertilizer and phosphorien}

An appreciable variation in number of grains/ row when maize plants carried one or two ears/ plant due to phosphorus fertilizer and phosphorien was detected in both seasons and the combined analysis (Table 3). Acquriment of the enlargement this numbers was the amenability of the application of phosphorien only when maize plants carried one ear/plant in the two seasons and their combined. But, when maize plant carried two ears/plant dualist application of 15 $\mathrm{kg} \mathrm{P}_{2} \mathrm{O}_{5} / \mathrm{fad}$., and phosphorien produced higher number of grains/row in the first season and combined data. The significant positive impact of chemical phosphorus fertilizer on number on grains/row was affirmed by Iqbal and Chauhan (2003) and Khan et al. (2005).

The stimulant effect of phosphoru, biofertilizers on number of grains/row was recorded by many workers such as Chabot et al. (1993).

\section{Interaction effect}

SC 167 hybrid appeared to produce higher number of grains/row when maize plants carried one or two ears/ plant under different $\mathrm{N}$ Levels with exception under $90 \mathrm{Kg} \mathrm{N} /$ fad., in mono eared plants followed by SC 168 while TWC 352 showed lower number of grains/row. while, under $90 \mathrm{~kg} \mathrm{~N} / \mathrm{fad}$., on one hand, number of grains/row of SC 167 and TWC 352 were higher when plants carried one or two ears/plant followed by $120 \mathrm{Kg} \mathrm{N} / \mathrm{fad}$., for TWC 352 in this connection (Table 3-a).

Regarding maize plants carried one ear/plant, in general, under both phosphorien alone or 15 $\mathrm{kg} \mathrm{P}_{2} \mathrm{O}_{5} /$ fad., + phosphorien, the superior hybrid was SC 168 and followed by SC 167 and meantime TWC 352 showed the lowest number, while under, $30 \mathrm{Kg} \mathrm{P}_{2} \mathrm{O}_{5} /$ fad., SC 167 was the superior hybrid in this number followed by SC 168 and TWC 352, when maize plants carried two ears/plant, the superior hybrid was SC 167 under the three phosphorus treatments, meantime TWC 352 showed the lowest number (Table 3-b).

Results exhibited significant interaction effect (Table 3-c) between $\mathrm{N}$ fertilizer and phosphorus levels and phosphorien, the application of $90 \mathrm{Kg}$ $\mathrm{N} /$ fad., under any phosphorus fertilizer regime used, produced the largest number of grains/row when maize plant carried one or two ears/plant. The uppermost number of gains/row (47.06 g) was obtained under addition of $90 \mathrm{~kg} \mathrm{~N} / \mathrm{fad}$, and $15 \mathrm{Kg} \mathrm{P}_{2} \mathrm{O}_{5} /$ fad., + phosphorien, while the lowest number of grains/row (44.17 g) was attained due to addition both $60 \mathrm{~kg} \mathrm{~N} /$ fad and 15 $\mathrm{kg} \mathrm{P}_{2} \mathrm{O}_{5}+$ phosphorien in mono eared plants, this was also true when maize plant carried two ears/plant.

\section{Number of Rows Ear}

Results pertaining to number of rows/ear of three yellow maize when carried one or two ears/plant as influenced by both $\mathrm{N}$ and $\mathrm{P}$ fertilization as well as addition of phosphorien are presented in Table 4.

\section{Maize hybrid differences}

As shown in Table 4, maize hybrids show significant difference in number of rows/ear in maize mono eared plants, but without significant difference among the three yellow maize on this number in douple eared plants in the two seasons, and their combined, TWC 352 gave the highest values for number of grains/row, whereas the other two hubrids were equal in this number. These results are in line with those reported by Hejazi and Soleymain (2014) and Sorkhi and Fateh (2014).

\section{Nitrogen level effect}

Significant differences in number of rows/ear due to nitrogen fertilization treatments were observed in both seasons and their combined analysis when maize plants carried one or two ears/plant. But this addition was without significant effect in the first season on the douple eared plants/plant. In mono eared plants, the first and second nitrogen increment failed to increase number of rows lear, while this character responded to $120 \mathrm{~kg} \mathrm{~N} / \mathrm{fad}$., which recorded the highest number of rows/ear. But, in maize douple eared plants, $90 \mathrm{~kg} \mathrm{~N} / \mathrm{fad}$., was quite enough to produce the largest averages of number of rows/ear in the second season and the combined (Table 4). the obtained data, also confirm the idea that applied by Abd ElMaksoud and Sarhan (2008), Atiaa et al. (2009), Abdou et al. (2012), Mukhtar et al. (2012) and El-Sobky (2014). 
Table 3-a. Effect of interaction between $\mathrm{N}$ fertilizer levels and maize hybrids on number of grains/row (combined data)

\begin{tabular}{lcccc}
\hline Maize hybrid (H) & \multicolumn{4}{c}{ N level (kg N/fad.) } \\
\cline { 2 - 5 } & $\mathbf{3 0}$ & $\mathbf{6 0}$ & $\mathbf{9 0}$ & $\mathbf{1 2 0}$ \\
\hline & $\mathrm{D}$ & $\mathrm{M}$ Mono eared plants & \\
SC 168 & $\mathrm{C}$ & $\mathrm{A}$ & $\mathrm{B}$ \\
& $46.89 \mathrm{~b}$ & $48.28 \mathrm{a}$ & $49.83 \mathrm{a}$ & $49.06 \mathrm{~b}$ \\
SC 167 & $\mathrm{C}$ & $\mathrm{B}$ & $\mathrm{A}$ & $\mathrm{A}$ \\
& $47.33 \mathrm{a}$ & $48.56 \mathrm{a}$ & $49.22 \mathrm{~b}$ & $49.5 \mathrm{a}$ \\
TWC 352 & $\mathrm{B}$ & $\mathrm{D}$ & $\mathrm{A}$ & $\mathrm{C}$ \\
& $40.67 \mathrm{c}$ & $38.11 \mathrm{~b}$ & $41.05 \mathrm{c}$ & $38.56 \mathrm{c}$ \\
& & Douple eared plants & \\
SC 168 & 30 & 60 & 90 & 120 \\
& $\mathrm{~B}$ & $\mathrm{~A}$ & $\mathrm{~A}$ & $\mathrm{~A}$ \\
SC 167 & $43.83 \mathrm{~b}$ & $44.94 \mathrm{a}$ & $45.06 \mathrm{~b}$ & $45.5 \mathrm{a}$ \\
& $\mathrm{BC}$ & $\mathrm{C}$ & $\mathrm{A}$ & $\mathrm{B}$ \\
TWC 352 & $45.22 \mathrm{a}$ & $44.89 \mathrm{a}$ & $46.89 \mathrm{a}$ & $45.67 \mathrm{a}$ \\
& $\mathrm{B}$ & $\mathrm{D}$ & $\mathrm{A}$ & $\mathrm{C}$ \\
& $40.11 \mathrm{c}$ & $36.39 \mathrm{~b}$ & $41.22 \mathrm{c}$ & $37.17 \mathrm{~b}$ \\
\hline
\end{tabular}

Table 3-b. Effect of interaction between maize hybrids and phosphorus fertilization regimes on number of grains/ row (combined data)

\begin{tabular}{|c|c|c|c|}
\hline \multirow[t]{2}{*}{ Maize hybrids (H) } & \multicolumn{3}{|c|}{ Phosphorus fertilization regimes } \\
\hline & $30 \mathrm{~kg} \mathrm{P}_{2} \mathrm{O}_{5} / \mathrm{fad}$. & Phosphorien & $15 \mathrm{~kg} \mathrm{P}_{2} \mathrm{O}_{5} /$ fad. + phosphorien \\
\hline & \multicolumn{3}{|c|}{ Mono eared plants } \\
\hline & $\mathbf{C}$ & $\mathbf{B}$ & $\mathbf{A}$ \\
\hline \multirow[t]{2}{*}{ SC 168} & $47.95 b$ & $48.54 \mathrm{a}$ & $49.04 \mathrm{a}$ \\
\hline & $\mathrm{B}$ & A & A \\
\hline \multirow[t]{2}{*}{ SC 167} & $48.25 \mathrm{a}$ & $48.75 \mathrm{a}$ & $48.96 \mathrm{a}$ \\
\hline & B & A & $\mathrm{C}$ \\
\hline \multirow[t]{3}{*}{ TWC 352} & $39.54 \mathrm{c}$ & $40.67 \mathrm{~b}$ & $38.58 b$ \\
\hline & \multicolumn{3}{|c|}{ Douple eared plants } \\
\hline & $\mathrm{C}$ & B & A \\
\hline \multirow[t]{2}{*}{ SC 168} & $43.67 \mathrm{a}$ & $44.67 \mathrm{~b}$ & $46.17 b$ \\
\hline & $\mathrm{C}$ & B & A \\
\hline \multirow[t]{2}{*}{ SC 167} & $43.63 \mathrm{a}$ & $45.92 \mathrm{a}$ & $47.46 \mathrm{a}$ \\
\hline & A & A & A \\
\hline TWC 352 & $38.83 b$ & $38.88 \mathrm{c}$ & $38.46 \mathrm{c}$ \\
\hline
\end{tabular}


Zagazig J. Agric. Res., Vol. 45 No. (5) 2018

Table 3-c. Effect of interaction between $\mathbf{N}$ fertilizer levels and phosphorus fertilization regimes on number of grains/row (combined data)

\begin{tabular}{|c|c|c|c|c|}
\hline \multirow[t]{2}{*}{ Phosphorus fertilization regimes } & \multicolumn{4}{|c|}{ N levels (kg N/fad.) } \\
\hline & 30 & 60 & 90 & 120 \\
\hline & \multicolumn{4}{|c|}{ Mono eared plants } \\
\hline \multirow[t]{2}{*}{$30 \mathrm{~kg} \mathrm{P}_{2} \mathrm{O}_{5} /$ fad. } & $\mathrm{D}$ & $\mathrm{c}$ & A & B \\
\hline & $44.56 \mathrm{c}$ & $44.83 b$ & $46.11 b$ & $45.5 b$ \\
\hline \multirow[t]{2}{*}{ Phosphorien } & $\mathrm{C}$ & $\mathrm{BC}$ & A & B \\
\hline & $44.89 \mathrm{~b}$ & $45.94 \mathrm{a}$ & $46.94 a$ & $46.17 \mathrm{a}$ \\
\hline \multirow[t]{4}{*}{ 15kg $\mathrm{P}_{2} \mathrm{O}_{5} /$ fad. + phosphorien } & B & $\mathrm{C}$ & A & B \\
\hline & $45.44 \mathrm{a}$ & $44.17 \mathrm{c}$ & $47.06 \mathrm{a}$ & $45.44 b$ \\
\hline & \multicolumn{4}{|c|}{ Douple eared plants } \\
\hline & A & A & A & $\mathrm{B}$ \\
\hline \multirow[t]{2}{*}{$30 \mathrm{~kg} \mathrm{P}_{2} \mathrm{O}_{5} / \mathrm{fad}$} & $42.5 \mathrm{~b}$ & $42.17 \mathrm{ab}$ & $42.61 \mathrm{c}$ & $40.89 c$ \\
\hline & $\mathrm{C}$ & $\mathrm{BC}$ & A & B \\
\hline \multirow[t]{2}{*}{ Phosphorien } & $42.33 b$ & $42.61 \mathrm{a}$ & $44.56 b$ & $43.17 \mathrm{~b}$ \\
\hline & B & $\mathrm{C}$ & A & B \\
\hline $15 \mathrm{~kg} \mathrm{P}_{2} \mathrm{O}_{5}+$ phosphorien & $44.33 \mathrm{a}$ & $41.44 \mathrm{~b}$ & $46.06 \mathrm{a}$ & $44.28 \mathrm{a}$ \\
\hline
\end{tabular}

Table 4. Number of rows/ear of maize as influenced by the different treatments during 2013 and 2014 seasons

\begin{tabular}{|c|c|c|c|c|c|c|}
\hline \multirow[t]{2}{*}{ Main effects and interactions } & \multicolumn{3}{|c|}{ Mono eared plants } & \multicolumn{3}{|c|}{ Douple eared plants } \\
\hline & 2013 & 2014 & Combined & 2013 & 2014 & Combined \\
\hline \multicolumn{7}{|l|}{ Maize hybrid (H) } \\
\hline SC 168 & $15.78 b$ & $16.00 \mathrm{~b}$ & $19.89 b$ & 19.89 & 16.33 & 16.11 \\
\hline SC 167 & $15.89 b$ & $15.89 b$ & $19.89 b$ & 19.83 & 16.39 & 16.11 \\
\hline TWC 352 & $16.61 \mathrm{a}$ & $16.61 \mathrm{a}$ & $16.61 \mathrm{a}$ & 16.11 & 16.39 & 16.25 \\
\hline F. test & $* *$ & $* *$ & $* *$ & NS & NS & NS \\
\hline \multicolumn{7}{|l|}{ Nitrogen level (kg N/fad.) (N) } \\
\hline 30 & $15.85 b$ & $16.00 \mathrm{~b}$ & 15.93 & 15.93 & $16 \mathrm{~b}$ & $15.96 \mathrm{~b}$ \\
\hline 60 & $15.93 b$ & $16.07 b$ & $16 b$ & 16.00 & $15.93 b$ & $15.96 \mathrm{~b}$ \\
\hline 90 & $16.07 b$ & $16 b$ & $16.04 \mathrm{~b}$ & 16.00 & $16.81 \mathrm{a}$ & $16.41 \mathrm{a}$ \\
\hline 120 & $16.52 \mathrm{a}$ & $16.59 \mathrm{a}$ & $16.56 \mathrm{a}$ & 15.85 & $16.74 \mathrm{a}$ & $16.30 \mathrm{a}$ \\
\hline F. test & $* *$ & $* *$ & $* *$ & NS & $* *$ & $* *$ \\
\hline \multicolumn{7}{|c|}{ Phosphorus fertilization regimes (p) } \\
\hline $30 \mathrm{~kg} \mathrm{P}_{2} \mathrm{O}_{5} / \mathrm{fad}$ & $16.11 \mathrm{ab}$ & $16.11 b$ & $16.11 b$ & 15.94 & $15.94 b$ & $15.94 b$ \\
\hline phosphorien & $15.89 \mathrm{~b}$ & $15.89 b$ & $15.89 \mathrm{C}$ & 16.00 & $16.67 \mathrm{a}$ & $16.33 \mathrm{a}$ \\
\hline $15 \mathrm{~kg} \mathrm{P}_{2} \mathrm{O}_{5} /$ fad.+ phosphorien & $16.28 \mathrm{a}$ & $16.50 \mathrm{a}$ & $16.39 \mathrm{a}$ & 15.89 & $16.5 \mathrm{a}$ & $16.19 \mathrm{a}$ \\
\hline F. test & $* *$ & $* *$ & $* *$ & NS & $* *$ & $* *$ \\
\hline \multicolumn{7}{|l|}{ Interactions } \\
\hline $\mathbf{H} \times \mathbf{N}$ & NS & ** & ** & NS & ** & $*$ \\
\hline $\mathbf{H} \times \mathbf{P}$ & $*$ & NS & ** & NS & ** & $* *$ \\
\hline $\mathbf{N} \times \mathbf{P}$ & $* *$ & $* *$ & $* *$ & NS & $* *$ & $* *$ \\
\hline
\end{tabular}




\section{Phosphorus fertilizer and phosphorien}

Significant differences in number of rows/ear due to phosphorus fertilizer and addition of phosphorien were observed in the two seasons and their combined when maize plants carried one or two ears/plant with the exception of the first season in douple eared plants. Treated plants with $15 \mathrm{~kg} \quad \mathrm{P}_{2} \mathrm{O}_{5} /$ fad., + phosphorien ranked first in this number and addition of $30 \mathrm{~kg}$ $\mathrm{P}_{2} \mathrm{O}_{5} /$ fad., came in the second rank, but addition of phosphorien only came in the third rank in mono eared plants. But when maize plant carried two ears/plant, the plants, that received phosphorien only or $15 \quad \mathrm{~kg} \quad \mathrm{P}_{2} \mathrm{O}_{5} /$ fad., + phosphorien recorded the highest number of rows/ear without significant differences between them in the second season and the combined. The obtained results, also confirm the idea that applied by Ahmad et al. (2007), Hussain et al. (2007) and Mukhtar et al. (2012).

\section{Interaction effect}

Under $30 \mathrm{~kg} \mathrm{~N} /$ fad., in maize mono eared plants and under 30 or $90 \mathrm{~kg} \mathrm{~N} / \mathrm{fad}$., in douple eared plants, each of SC 168, SC 167 and TWC 352 had equal number of rows/ear. But under 90 or $120 \mathrm{~kg} \mathrm{~N} /$ fad., in mono eared plants and 60 $\mathrm{Kg} \mathrm{N} /$ fad., in douple eared plants. TWC $352 \mathrm{had}$ higher number of rows/ear than each of SC 168 and SC 167. For the three yellow maize hubrids in mono eared plants, $120 \mathrm{~kg} \mathrm{~N} /$ fad., gave higher number of rows/ear than the other $\mathrm{N}$ treatment, but in douple eared plants $90 \mathrm{~kg} \mathrm{~N} / \mathrm{fad}$., was quite enough to produce the highest averages of this number in the three yellow moize hybrids (Table 4-a).

Under $30 \mathrm{~kg} \mathrm{P}_{2} \mathrm{O}_{5} /$ fad., in maize mono eared plants and under each of phosphorien or $15 \mathrm{~kg}$ $\mathrm{P}_{2} \mathrm{O}_{5} /$ fad., + phosphorien in douple eared plants, the three yellow maize had equal number of rows/ear. But under each of phosphorien and 15 $\mathrm{kg} \mathrm{P}_{2} \mathrm{O}_{5} /$ fad., + phosphorien in mono eared plants and under $30 \mathrm{~kg} \mathrm{P}_{2} \mathrm{O}_{5} /$ fad., in douple eared plants, TWC 352 had higher number of rows/ear than each of SC 168 and SC 167 but, for TWC 352 the largest number of rows/ear was obtained from applying $15 \mathrm{~kg} \mathrm{P}_{2} \mathrm{O}_{5} /$ fad., + phosphorien and the other two phosphorus treatments gave the highest value under phosphorien treatment in douple eared plants (Table 4-b).
Under $30 \mathrm{~kg} \mathrm{~N} /$ fad., in mono eared plants and under both 30 and $90 \mathrm{Kg} \mathrm{N} /$ fad., in douple eared plants, the three phosphorus treatments had, equal number of rows/ear. But under 60 and $120 \mathrm{~kg} \mathrm{~N} /$ fad., in mono eared plants and 120 $\mathrm{kg} \mathrm{N} /$ fad., in douple eared plants, maize plant fertilized by $15 \mathrm{~kg} \mathrm{P}_{2} \mathrm{O}_{5} /$ fad., + phosphorien produced higher number of rows/ear. When maize plants fertilized by $30 \mathrm{~kg} \mathrm{P}_{2} \mathrm{O}_{5} / \mathrm{fad}$., in mono eared plants, $90 \mathrm{~kg} \mathrm{~N} /$ fad., was quite enough to produce the highest averages of this number, but when fertilized by phosphorien only in douple eared plants, $60 \mathrm{~kg} \mathrm{~N} / \mathrm{fad}$., was quite in this respect (Table 4-c).

\section{Hundred Grain Weight (g)}

\section{Maize hybrids differences}

Results in Table 5 confirm high significant differences among the three hybrids in hundred grain weight when maize plants carried one or two ears/plant. The combined analysis results indicated that SC 167 produced the heaviest hundred grain weight followed by SC 168 while, TWC 352 gave the lighter hundred grain weight. This picture is clearly true in the first season and combined in mono eared plants and also the two seasons beside their combined when maize plants carried two ears/ plant. These results are in line with those reported by Sharifi et al. (2009), Golezani and Tiajbakhsh (2012), Radma and Dagash (2013), Kandil (2013) and Modhej et al. (2014).

\section{Nitrogen level effect}

Statistical analysis revealed highly significant differences among nitrogen fertilizer levels in both seasons and their combined when maize plants carried one or two ears/plant. Meanwhile, appliance of 30 or $120 \mathrm{~kg} \mathrm{~N} / \mathrm{fad}$., produced the heaviest weight of 100 grains followed by both 60 and $90 \mathrm{~kg} \mathrm{~N} /$ fad., in mono eared plants, whereas in maize douple eared plants, appliance of $30 \mathrm{~kg} \mathrm{~N} / \mathrm{fad}$., produced the heaviest 100 grain weight followed by both 90 and $120 \mathrm{~kg} \mathrm{~N} /$ fad., but the lowest weight was obtained from applying $60 \mathrm{~kg} \mathrm{~N} /$ fad., (combined data).

The positive effect of $\mathrm{N}$ feltilizer on hundred grain weight was reported by El-Sobky (2014), Sorkhi and Fateh (2014), El-Kholy (2015) and Ikramullah et al. (2015). 
Table 4-a. Effect of interaction between $\mathbf{N}$ fertilizer levels and maize hybrids on number of rows/ear (combined data)

\begin{tabular}{|c|c|c|c|c|}
\hline \multirow[t]{2}{*}{ Maize hybrid (H) } & \multicolumn{4}{|c|}{ N levels (kg N/fad.) } \\
\hline & 30 & 60 & 90 & 120 \\
\hline & \multicolumn{4}{|c|}{ Mono eared plants } \\
\hline & B & $\mathrm{B}$ & $\mathrm{B}$ & A \\
\hline \multirow[t]{2}{*}{ SC 168} & $15.89 \mathrm{a}$ & $15.78 \mathrm{~b}$ & $15.67 \mathrm{~b}$ & $16.22 b$ \\
\hline & $\mathrm{AB}$ & $\mathrm{B}$ & $\mathrm{B}$ & A \\
\hline \multirow[t]{2}{*}{ SC 167} & $15.89 \mathrm{a}$ & $15.78 \mathrm{a}$ & $15.78 b$ & $16.11 \mathrm{~b}$ \\
\hline & $\mathrm{C}$ & B & B & A \\
\hline \multirow[t]{3}{*}{ TWC 352} & $16.00 \mathrm{a}$ & $16.44 \mathrm{a}$ & $16.67 \mathrm{a}$ & $17.33 \mathrm{a}$ \\
\hline & \multicolumn{4}{|c|}{ Douple eared plants } \\
\hline & B & $\mathrm{B}$ & A & A \\
\hline \multirow[t]{2}{*}{ SC 168} & $15.89 \mathrm{a}$ & $15.78 \mathrm{~b}$ & $16.33 \mathrm{a}$ & $16.44 \mathrm{a}$ \\
\hline & $\mathrm{B}$ & $\mathrm{B}$ & A & A \\
\hline \multirow[t]{2}{*}{ SC 167} & $15.89 \mathrm{a}$ & $15.78 \mathrm{~b}$ & $16.33 \mathrm{a}$ & $16.44 \mathrm{a}$ \\
\hline & B & $\mathrm{AB}$ & A & B \\
\hline TWC 352 & $16.11 \mathrm{a}$ & $16.33 \mathrm{a}$ & $16.56 \mathrm{a}$ & $16.00 \mathrm{~b}$ \\
\hline
\end{tabular}

Table 4-b. Effect of interaction between maize hybrids and phosphorus fertilization regimes on number of rows/ ear (combined data)

\begin{tabular}{|c|c|c|c|}
\hline \multirow[t]{2}{*}{ Maize hybrid (H) } & \multicolumn{3}{|c|}{ Phosphorus fertilization regimes } \\
\hline & $30 \mathrm{~kg} \mathrm{P}_{2} \mathrm{O}_{5} /$ fad. & Phosphorien & $15 \mathrm{~kg} \mathrm{P}_{2} \mathrm{O}_{5} /$ fad. + phosphorien \\
\hline & \multicolumn{3}{|c|}{ Mono eared plants } \\
\hline & A & A & A \\
\hline \multirow[t]{2}{*}{ SC 168} & $16.00 \mathrm{a}$ & $15.67 b$ & $16.00 \mathrm{~b}$ \\
\hline & $\mathrm{AB}$ & B & A \\
\hline \multirow[t]{2}{*}{ SC 167} & $16.00 \mathrm{a}$ & $15.50 \mathrm{~b}$ & $16.17 b$ \\
\hline & B & B & A \\
\hline \multirow[t]{3}{*}{ TWC 352} & $16.00 \mathrm{a}$ & $16.50 \mathrm{a}$ & $17.00 \mathrm{a}$ \\
\hline & \multicolumn{3}{|c|}{ Douple eared plants } \\
\hline & A & $\mathrm{B}$ & $\mathrm{B}$ \\
\hline \multirow[t]{2}{*}{ SC 168} & $15.75 b$ & $16.33 \mathrm{a}$ & $16.25 \mathrm{a}$ \\
\hline & B & A & A \\
\hline \multirow[t]{2}{*}{ SC 167} & $15.75 b$ & $16.25 \mathrm{a}$ & $16.33 \mathrm{a}$ \\
\hline & $\mathrm{AB}$ & A & B \\
\hline TWC 352 & $16.33 \mathrm{a}$ & $16.42 \mathrm{a}$ & $16.00 \mathrm{a}$ \\
\hline
\end{tabular}


Table 5. Hundred grain weight (g) of maize as influenced by the different treatments during 2013 and 2014 seasons

\begin{tabular}{|c|c|c|c|c|c|c|}
\hline \multirow[t]{3}{*}{ Main effects and interactions } & \multicolumn{6}{|c|}{ Hundred grain weight (g) } \\
\hline & \multicolumn{3}{|c|}{ Mono eared plants } & \multicolumn{3}{|c|}{ Douple eared plants } \\
\hline & 2013 & 2014 & Combined & 2013 & 2014 & Combined \\
\hline \multicolumn{7}{|l|}{ Moize hybrid (H) } \\
\hline SC 168 & $38.47 \mathrm{~b}$ & $39.85 \mathrm{a}$ & $39.16 b$ & $36.4 \mathrm{~b}$ & $37.10 \mathrm{~b}$ & $36.70 \mathrm{~b}$ \\
\hline SC 167 & $39.69 \mathrm{a}$ & $40.39 a$ & $40.04 \mathrm{a}$ & $37.86 \mathrm{a}$ & $38.80 \mathrm{a}$ & $38.30 \mathrm{a}$ \\
\hline TWC 352 & $37.59 \mathrm{c}$ & $37.31 b$ & $37.45 \mathrm{c}$ & $35.20 \mathrm{c}$ & $36.10 \mathrm{c}$ & $35.70 \mathrm{c}$ \\
\hline F. test & $* *$ & $* *$ & $* *$ & $* *$ & $* *$ & $* *$ \\
\hline \multicolumn{7}{|l|}{ Nitrogen level (kg N/fad.) (N) } \\
\hline 30 & $40.08 \mathrm{a}$ & $39.48 \mathrm{ab}$ & $39.78 \mathrm{a}$ & $38.00 \mathrm{a}$ & $38.70 \mathrm{a}$ & $38.30 \mathrm{a}$ \\
\hline 60 & $36.59 \mathrm{~d}$ & $37.31 b$ & $36.95 b$ & $35.20 \mathrm{~d}$ & $35.70 \mathrm{c}$ & $35.50 \mathrm{c}$ \\
\hline 90 & $38.16 \mathrm{c}$ & $38.77 \mathrm{ab}$ & $38.46 b$ & $36.50 \mathrm{~b}$ & $37.40 \mathrm{~b}$ & $36.90 \mathrm{~b}$ \\
\hline 120 & $39.74 b$ & $41.17 \mathrm{a}$ & $40.46 \mathrm{a}$ & $36.20 \mathrm{c}$ & $37.60 \mathrm{~b}$ & $36.90 b$ \\
\hline F.test & $* *$ & $* *$ & $* *$ & $* *$ & $* *$ & $* *$ \\
\hline \multicolumn{7}{|c|}{ Phosphorus fertilization regimes (p) } \\
\hline $30 \mathrm{~kg} \mathrm{P}_{2} \mathrm{O}_{5} /$ fad. & $36.88 \mathrm{~b}$ & $38.34 \mathrm{~b}$ & $37.61 b$ & $37.50 \mathrm{~b}$ & $38.10 \mathrm{~b}$ & $37.80 \mathrm{~b}$ \\
\hline phosphorien & $36.79 b$ & $37.65 b$ & $37.22 b$ & $33.20 \mathrm{c}$ & $34.30 \mathrm{c}$ & $33.70 \mathrm{c}$ \\
\hline $15 \mathrm{~kg} \mathrm{P}_{2} \mathrm{O}_{5} /$ fad. + phosphorien & $42.26 \mathrm{a}$ & $41.26 \mathrm{a}$ & $41.91 \mathrm{a}$ & $38.80 \mathrm{a}$ & $39.60 \mathrm{a}$ & $39.20 \mathrm{a}$ \\
\hline F. test & $* *$ & $* *$ & $* *$ & $* *$ & $* *$ & $* *$ \\
\hline \multicolumn{7}{|l|}{ Interactions } \\
\hline $\mathbf{H} \times \mathbf{N}$ & $* *$ & NS & $* *$ & $* *$ & $* *$ & $* *$ \\
\hline $\mathbf{H} \times \mathbf{P}$ & $* *$ & $* *$ & $* *$ & $* *$ & $* *$ & $* *$ \\
\hline $\mathbf{N} \times \mathbf{P}$ & $* *$ & $* *$ & $* *$ & $* *$ & $* *$ & $* *$ \\
\hline
\end{tabular}

\section{Phosphorus fertilizer and phosphorien}

An appreciable variation in hundred grain weight when maize plants carried one or two eared/plant due to phosphorus fertilizer and phosphorien was detected in both seasons and their combined (Table 5). Acquirement of the heaviest grains was via the application of $15 \mathrm{~kg}$ $\mathrm{P}_{2} \mathrm{O}_{5} /$ fad., + phosphoien, while the lowest hundred grain weight was given by each of 30 $\mathrm{kg} \mathrm{P}_{2} \mathrm{O}_{5} /$ fad., or phosphorien only in both seasons and their combined when maize plants carried one ear/plant. This picture is true also in the douple eared plants, but addition of phosphorien only gave the lowest hundred weight in the two seasons and their combined.

The significant positive impact of chemical phosphorus fertilizer on 100 grain weight was affirmed by Ahmad et al. (2007) and Omar (2011).

\section{Interaction effect}

Under 30and $90 \mathrm{~kg} \mathrm{~N} /$ fad., in maize mono eared plants and also, under $60 \mathrm{~kg} \mathrm{~N} /$ fad., in douple eared plants, each of SC 168 and SC 167 had the heaveiest hundred grain weight. But under the same level of $\mathrm{N}$ (30 and 90) in mono eared plants and under $60 \mathrm{~kg} \mathrm{~N} / \mathrm{fad}$., in douple eared plants, TWC 352 gave the lightest hundred grain weight. No clear trend could be detected for this character in this respect (Table 5-a).

Concerning mono eared plants, under $30 \mathrm{~kg}$ $\mathrm{P}_{2} \mathrm{O}_{5} /$ fad., both SC 168 and TWC 352 had the heaviest 100 grain weight while SC 167 gave the lightest weight, but under each of sole phosphorien 
Table 5-a. Effect of interaction between $\mathrm{N}$ fertilizer levels and maize hybrids on hundred grain weight (combined data)

\begin{tabular}{lcccc}
\hline Maize hybrid (H) & \multicolumn{4}{c}{ N levels Kg N/fad. } \\
\cline { 2 - 5 } & $\mathbf{3 0}$ & $\mathbf{6 0}$ & $\mathbf{9 0}$ & $\mathbf{1 2 0}$ \\
\hline & $\mathrm{A}$ & $\mathrm{B}$ & Mono eared plants & $\mathrm{A}$ \\
SC 168 & $40.57 \mathrm{a}$ & $35.46 \mathrm{~b}$ & $39.54 \mathrm{a}$ & $41.07 \mathrm{a}$ \\
& $\mathrm{A}$ & $\mathrm{A}$ & $\mathrm{A}$ & $\mathrm{B}$ \\
SC 167 & $40.81 \mathrm{a}$ & $38.08 \mathrm{a}$ & $40.02 \mathrm{a}$ & $35.69 \mathrm{~b}$ \\
& $\mathrm{~A}$ & $\mathrm{AB}$ & $\mathrm{B}$ & $\mathrm{A}$ \\
TWC 352 & $37.96 \mathrm{~b}$ & $37.32 \mathrm{ab}$ & $35.83 \mathrm{~b}$ & $39.07 \mathrm{a}$ \\
& & & Douple eared plants & \\
SC 168 & $\mathrm{A}$ & $\mathrm{A}$ & $\mathrm{AB}$ & $\mathrm{B}$ \\
& $37.05 \mathrm{~b}$ & $37.07 \mathrm{a}$ & $36.51 \mathrm{~b}$ & $36.23 \mathrm{~b}$ \\
SC 167 & $\mathrm{A}$ & $\mathrm{C}$ & $\mathrm{D}$ & $\mathrm{B}$ \\
& $40.37 \mathrm{a}$ & $37.64 \mathrm{a}$ & $36.69 \mathrm{~b}$ & $38.69 \mathrm{a}$ \\
TWC 352 & $\mathrm{A}$ & $\mathrm{C}$ & $\mathrm{A}$ & $\mathrm{B}$ \\
& $37.54 \mathrm{~b}$ & $31.65 \mathrm{~b}$ & $37.63 \mathrm{a}$ & $35.91 \mathrm{~b}$ \\
\hline
\end{tabular}

application or $15 \mathrm{~kg} \mathrm{P}_{2} \mathrm{O}_{5} /$ fad., + phosphorien, SC 168 and 167 had the heaviest, whereas TWC 352 recorded the lightest weight. For SC 168 and 167 , the heaviest grains was achieved by $15 \mathrm{~kg} \mathrm{P}_{2} \mathrm{O}_{5} /$ fad., + phosphorien, while the lowest weight was given by $30 \mathrm{~kg} \mathrm{P}_{2} \mathrm{O}_{5} / \mathrm{fad}$.

Regarding douple eared plants under the three phosphorus treatments, SC 167 had the heaviest hundred grain weight, while the lowest weight was given by each of SC 168 and TWC 352. For SC 168 and SC 167, the heaviest grains recorded by applying $15 \mathrm{~kg} \mathrm{P}_{2} \mathrm{O}_{5} /$ fad., + phosphorien and the lowest weight was given by phosphorien only (Table 5-b).

When maize plants carried one ear/plant and under $30 \mathrm{~kg} \mathrm{~N} /$ fad., the three phosphorus treatments had equal hundred grain weight, while the heaviest hundred grain weight was achived by addition of $15 \mathrm{~kg} \mathrm{P}_{2} \mathrm{O}_{5}+$ phosphorien under the other $\mathrm{N}$ levels $(60,90$ and $120 \mathrm{~kg}$ $\mathrm{N} /$ fad.). In addition, when maize plants fertilized by $30 \mathrm{Kg} \mathrm{P} \mathrm{P}_{5} /$ fad., or phosphorien, $30 \mathrm{~kg} \mathrm{~N} /$ fad., was quite enough to produce the heaviest hundred grain weight, but when fertilized by 15 $\mathrm{kg} \mathrm{P}_{2} \mathrm{O}_{5} /$ fad., + phosphorien, the heaviest weight was obtained by applying $120 \mathrm{~kg} \mathrm{~N} /$ fad., (Table 5-c).

Regarding douple eared plants, under 30 and $60 \mathrm{~kg} \mathrm{~N} / \mathrm{fad}$., the heaviest hundred grain weight was given by addition of $15 \quad \mathrm{~kg} \quad \mathrm{P}_{2} \mathrm{O}_{5}+$ phosphorien. Then, the heaviest grains was recorded by $30 \mathrm{~kg} \mathrm{P}_{2} \mathrm{O}_{5} /$ fad., under each of 90 and $120 \mathrm{~kg} \mathrm{~N} /$ fad.,. While when maize fertilized by phosphorien only or $15 \mathrm{~kg} \mathrm{P}_{2} \mathrm{O}_{5} /$ fad., + phosphorien, $30 \mathrm{~kg} \mathrm{~N} /$ fad., was quite enough to produce the heaviest weight (Table 5-c)

\section{Ear Grain Weight (g)}

Results concerning the hybrids differences and the effect of $\mathrm{N}$ levels and phosphorus fertilizer as well as phosphorien on ear grain weight are given in Table 6 .

\section{Maize hybrid differences}

Maize hybrids revealed highly significant differences in ear weight, where SC 167 hybrid appeared to produce the heaviest ear grain weight throughout the second season and their 
Table 5-b. Effect of interaction between maize hybrids and phosphorus fertilization regimes on hundred grain weight (g) (Combined data)

\begin{tabular}{|c|c|c|c|}
\hline \multirow[t]{2}{*}{ Maize hybrid (H) } & \multicolumn{3}{|c|}{ Phosphorus fertilization regimes } \\
\hline & $30 \mathrm{Kg} \mathrm{P}_{2} \mathrm{O}_{5} /$ fad., & Phosphorien & $15 \mathrm{~kg} \mathrm{P}_{2} \mathrm{O}_{5} /$ fad.+ phosphorien \\
\hline & \multicolumn{3}{|c|}{ Mono eared plants } \\
\hline \multirow{2}{*}{ SC 168} & $\mathrm{~B}$ & $\mathrm{~B}$ & A \\
\hline & $36.95 \mathrm{a}$ & $34.21 \mathrm{ab}$ & $43.32 \mathrm{a}$ \\
\hline \multirow{2}{*}{ SC 167} & $\mathrm{C}$ & $\mathrm{B}$ & $\mathrm{A}$ \\
\hline & $33.48 b$ & $38.45 \mathrm{a}$ & $44.30 \mathrm{a}$ \\
\hline \multirow{3}{*}{ TWC 352} & A & $\mathrm{B}$ & A \\
\hline & $38.23 \mathrm{a}$ & $36.00 \mathrm{~b}$ & $38.38 b$ \\
\hline & \multicolumn{3}{|c|}{ Douple eared plants } \\
\hline \multirow{3}{*}{ SC 168} & $\mathrm{~B}$ & $\mathrm{C}$ & A \\
\hline & $37.53 b$ & $32.85 b$ & $39.76 b$ \\
\hline & $\mathrm{B}$ & $\mathrm{C}$ & A \\
\hline SC 167 & $38.43 \mathrm{a}$ & $35.11 \mathrm{a}$ & $41.51 \mathrm{a}$ \\
\hline \multirow{2}{*}{ TWC 352} & A & $\mathrm{C}$ & $\mathrm{B}$ \\
\hline & $37.41 \mathrm{~b}$ & $33.27 b$ & $36.36 c$ \\
\hline
\end{tabular}

Table 5-c. Effect of interaction between $\mathbf{N}$ fertilizer levels and phosphorus fertilization regimes on hundred grain weight $(\mathrm{g})$

\begin{tabular}{|c|c|c|c|c|}
\hline \multirow[t]{2}{*}{ Phosphorus fertilization regimes } & \multicolumn{4}{|c|}{ N levels (kg/fad.) } \\
\hline & 30 & 60 & 90 & 120 \\
\hline & \multicolumn{4}{|c|}{ Mono eared plants } \\
\hline \multirow{2}{*}{$30 \mathrm{~kg} \mathrm{P}_{2} \mathrm{O}_{5} / \mathrm{fad}$} & A & $\mathrm{B}$ & A & $\mathrm{B}$ \\
\hline & $39.61 \mathrm{a}$ & $33.49 \mathrm{c}$ & $37.72 b$ & $34.06 \mathrm{~b}$ \\
\hline \multirow{2}{*}{ Phosphorien } & A & $\mathrm{B}$ & $\mathrm{B}$ & $\mathrm{B}$ \\
\hline & $40.15 \mathrm{a}$ & $37.12 b$ & $35.88 \mathrm{~b}$ & $35.73 b$ \\
\hline \multirow{3}{*}{$15 \mathrm{~kg} \mathrm{P}_{2} \mathrm{O}_{5} /$ fad. + phosphorien } & $\mathrm{C}$ & $\mathrm{BC}$ & $\mathrm{B}$ & A \\
\hline & $39.58 \mathrm{a}$ & $40.24 a$ & $41.79 \mathrm{a}$ & $46.03 \mathrm{a}$ \\
\hline & \multicolumn{4}{|c|}{ Douple eared plants } \\
\hline \multirow{2}{*}{$30 \mathrm{~kg} \mathrm{P}_{2} \mathrm{O}_{5} / \mathrm{fad}$} & $\mathrm{C}$ & B & $\mathrm{A}$ & $\mathrm{B}$ \\
\hline & $36.20 \mathrm{~b}$ & $37.86 \mathrm{~b}$ & $39.06 \mathrm{a}$ & $38.03 \mathrm{a}$ \\
\hline \multirow{2}{*}{ phosphorien } & $\mathrm{A}$ & $\mathrm{C}$ & $\mathrm{B}$ & $\mathrm{B}$ \\
\hline & $36.53 b$ & $29.97 \mathrm{c}$ & $34.16 \mathrm{c}$ & $34.31 \mathrm{~b}$ \\
\hline \multirow{2}{*}{$15 \mathrm{~kg} \mathrm{P}_{2} \mathrm{O}_{5} /$ fad. + phosphorien } & A & B & $\mathrm{C}$ & B \\
\hline & $42.22 \mathrm{a}$ & $38.53 \mathrm{a}$ & $37.60 \mathrm{~b}$ & $38.49 \mathrm{a}$ \\
\hline
\end{tabular}


Table 6. Ear grain weight (mono eared plants) of maize as influenced by the different treatments during 2013 and 2014 seasons

\begin{tabular}{|c|c|c|c|}
\hline Main effects and interactions & 2013 & 2014 & Combined \\
\hline \multicolumn{4}{|l|}{ Maize hybrid (H) } \\
\hline SC 168 & $266.6 \mathrm{a}$ & $245.4 b$ & $256.0 \mathrm{~b}$ \\
\hline SC 167 & $257.4 \mathrm{~b}$ & $267.3 \mathrm{a}$ & $262.3 \mathrm{a}$ \\
\hline TWC 352 & 205.bc & $205.6 \mathrm{c}$ & $205.7 \mathrm{c}$ \\
\hline F. test & $* *$ & $* *$ & $* *$ \\
\hline \multicolumn{4}{|l|}{ Nitrogen level (kg N/fad.) (N) } \\
\hline 30 & $220.6 \mathrm{~d}$ & $242.1 b$ & $231.4 d$ \\
\hline 60 & 240.bc & $246.3 \mathrm{a}$ & $243.4 \mathrm{~b}$ \\
\hline 90 & $243.2 b$ & $239.6 \mathrm{c}$ & $241.4 \mathrm{c}$ \\
\hline 120 & $268.6 \mathrm{a}$ & $229.8 \mathrm{~d}$ & $249.2 \mathrm{a}$ \\
\hline F. test & $* *$ & $* *$ & $* *$ \\
\hline \multicolumn{4}{|c|}{ Phosphorus fertilization regimes (p) } \\
\hline $30 \mathrm{~kg} \mathrm{P}_{2} \mathrm{O}_{5} / \mathrm{fad}$ & $235.5 b$ & $245.0 \mathrm{~b}$ & $240.3 b$ \\
\hline Phosphorien & $247.5 \mathrm{a}$ & $222.4 \mathrm{c}$ & $234.9 \mathrm{c}$ \\
\hline $15 \mathrm{~kg} \mathrm{P}_{2} \mathrm{O}_{5} /$ fad. + phosphorien & $246.7 \mathrm{a}$ & $250.9 \mathrm{a}$ & $248.8 \mathrm{a}$ \\
\hline F. test & $* *$ & $* *$ & $* *$ \\
\hline \multicolumn{4}{|l|}{ Interactions } \\
\hline $\mathbf{H} \times \mathbf{N}$ & $* *$ & $* *$ & $* *$ \\
\hline $\mathbf{H} \times \mathbf{P}$ & $* *$ & $* *$ & $* *$ \\
\hline $\mathbf{N} \times \mathbf{P}$ & $* *$ & $* *$ & $* *$ \\
\hline
\end{tabular}

combined. Otherwise, TWC 352 hybrid gave the lowest ear grain weight in this respect. This result almost followed the same patterns of hundred grain weight. The differences in ear grain weight among the there maize hybrids might be attributed to the genetically variation. Similar maize hybrids change in ear weight was obtained by Abdou et al. (2012).

\section{Nitrogen levels effect}

Nitrogen fertilization results revealed highly significant differences throughout the two seasons and their combined. Meanwhile, appliance of $120 \mathrm{~kg} \mathrm{~N} / \mathrm{fad}$., produced the heaviest ear grain weight followed by 60 and $90 \mathrm{~kg} \mathrm{~N} /$ fad., whereas the lowest weight of ear grain weight was obtained from $30 \mathrm{~kg} \mathrm{~N} /$ fad., (combined data).
(Table 6) Several investigators came to the same conclusion such as El-Moursy (2013), Kandil (2013), Hejazi and Soleymain (2014) and Khan et al. (2014).

\section{Phosphorus fertilizer and phosphorien effect}

Significant differences in ear grain weight due to phosphorus fertilizer and addition of phosphorien were observed in the two seasons and their combined. Treated plants with $15 \mathrm{~kg}$ $\mathrm{P}_{2} \mathrm{O}_{5} /$ fad., + phosphorien ranked first in this weight and addition of $30 \mathrm{~kg} \mathrm{P}_{2} \mathrm{O}_{5} /$ fad., came in the second rank, but addition of phosphorien only came in the last rank, this was true in the second season and the combined (Table 6). Several authors came to the same conclusion of 
them Yosefi et al. (2011), Zafar et al. (2011) and Khan et al. (2014). In addition Karimian (2000) and Jat and Shaktawat (2003) confirmed satisfactory results from the use of phosphate biological fertilizer.

\section{Interaction effect}

Under 30 and $120 \mathrm{~kg} \mathrm{~N} /$ fad., SC 167 had the heaviest ear grain weight followed by SC 168, where under 60 and $90 \mathrm{~kg} \mathrm{~N} /$ fad., SC 168 recorded the heaviest ear grain weight, while TWC 352 gave the lowest weight under the four $\mathrm{N}$ level. Therefore, the highest ear grain weight (287 g) was achieved by SC 167 hybrid when $120 \mathrm{~kg} \mathrm{~N} /$ fad., was applied. Otherwise, TWC 352 hybrid gave the lowest ear grain weight (201 g) under applying $90 \mathrm{~kg} \mathrm{~N} /$ fad., (Table 6-a).

Under 60 and $120 \mathrm{~kg} \mathrm{~N} /$ fad., the heaviest ear grain weight was recorded when maize plants fertilized by $15 \mathrm{~kg} \mathrm{P}_{2} \mathrm{O}_{5}+$ phosphorien, but recoded by $90 \mathrm{~kg} \mathrm{~N} /$ fad., when fertilized by 30 $\mathrm{kg} \mathrm{P}_{2} \mathrm{O}_{5} / \mathrm{fad}$. When maize plants fertilized with phosphorien only, $30 \mathrm{~kg} \mathrm{~N} / \mathrm{fad}$, was quite enough to produce the heaviest ear grain weight, but when fertilized by $30 \mathrm{~kg} \mathrm{P}_{2} \mathrm{O}_{5} /$ fad., or $15 \mathrm{~kg}$ $\mathrm{P}_{2} \mathrm{O}_{5}+$ phosphorien they were needed 90 and $120 \mathrm{~kg} \mathrm{~N} /$ fad., respectively. Then, the highest ear grain weight (264.5) was recorded when maize plants fertilized by each of $15 \mathrm{~kg} \mathrm{P}_{2} \mathrm{O}_{5} /$ fad., + phosphorien and $120 \mathrm{~kg} \mathrm{~N} / \mathrm{fad}$, where the lower ear grain weight (223.1) was recorded when fertilized by each of $30 \mathrm{~kg} \mathrm{P}_{2} \mathrm{O}_{5} /$ fad., and $30 \mathrm{~kg}$ N/fad., (Table 6-b).

Under $30 \mathrm{~kg} \mathrm{P}_{2} \mathrm{O}_{5} /$ fad., and $15 \mathrm{~kg} \mathrm{P}_{2} \mathrm{O}_{5} /$ fad., + phosphorien, $\mathrm{SC} 167$ recorded the highest ear grain weight, while, SC 168 gave the heaviest weight when maize plants were fertilized by phosphorien only, then the lowest ear grain weight was achieved by TWC 352 in all phosphorus fertilizer. Therefore, the heaviest ear grain weight of 277.8 was obtained by SC 167 hybrid when $15 \mathrm{~kg} \mathrm{P}_{2} \mathrm{O}_{5} /$ fad., + phosphorien were applied. Otherwise, TWC 352 gave the lowest ear grain weight (203 g) under phosphorien only applied (Table 6-c).

\section{Grain Yield (ardab/fad.)}

The average effects of nitrogen fertilizer levels and addition of phosphorus and phosphorien on grain yield of the three yellow maize hybrids are shown in Table 7.

\section{Maize hybrid differences}

Significant differences could be detected between the three yellow maize hybrids in the two seasons and their combined, where SC 167 hybrid had greater grain yield/fad., than the other maize hybrids. Otherwise, TWC 352 hybrid gave the lowest grain yield/fad., in this respect. This result almost followed the same patterns of hundred grain weight and ear grain weight. This inclination was fairly virtual in both growing seasons and affirmed when their data were statistically combined. The hybrid differences in such trait could be explained by the fact that photosynthetic translocate from the source to the sink were great enough to fill all the grains of SC 167 hybrid or to increase the final grain yield/fad., relative to TWC 352 or SC 168. These results are in conformity with those given by Abd El-Maksoud and Sarhan (2008), Atiaa et al. (2009), Abdou et al. (2012) and Mukhtar et al. (2012).

\section{Nitrogen levels effect}

It is evident from Table 7 that each $\mathrm{N}$ increment up to $120 \mathrm{~kg} \mathrm{~N} / \mathrm{fad}$, produced a significant increase in grain yield ( $\mathrm{arda} / \mathrm{fad}$.), in both seasons and their combined. The response of grain yield to the increase of $\mathrm{N}$ level reflected that observed in each of ear length, number of grains/row, number of rows/ear and ear grain weight (Tables 2, 3, 4 and 6) several authors reported significant increase in grain yield due to the increase of $\mathrm{N}$ level (Sharifi et al., 2009; Golezani and Tiajbakhsh, 2012; Kandil, 2013;Radma and Dagash, 2013; Modhej et al., 2014).

\section{Phosphorus fertilizer and phosphorien effect}

Significant differences in grain yield/fad., due to phosphorus fertilizer and addition of phosphorien were observed in the two seasons and their combined. Acquirement of the heaviest grain yield/fad., was by application of $30 \mathrm{~kg}$ $\mathrm{P}_{2} \mathrm{O}_{5} /$ fad., or phosphorien only, while the lowest grain yield/fad., was given by addition $15 \mathrm{~kg}$ $\mathrm{P}_{2} \mathrm{O}_{5} /$ fad., + phosphorien in both seasons and their combined (Table 7). 
Table 6-a. Effect of interaction between $N$ fertilizer levels and maize hybrids on ear grain weight (g) in mono eared plants (combined data)

\begin{tabular}{lcccc}
\hline Maize hybrid (H) & \multicolumn{4}{c}{ N levels (kg N/fad.) } \\
\cline { 2 - 5 } & $\mathbf{3 0}$ & $\mathbf{6 0}$ & $\mathbf{9 0}$ & $\mathbf{1 2 0}$ \\
\hline \multirow{2}{*}{ SC 168 } & $\mathrm{D}$ & $\mathrm{B}$ & $\mathrm{A}$ & $\mathrm{C}$ \\
& $239.9 \mathrm{~b}$ & $261.5 \mathrm{a}$ & $267.0 \mathrm{a}$ & $255.7 \mathrm{~b}$ \\
SC 167 & $\mathrm{D}$ & $\mathrm{B}$ & $\mathrm{C}$ & $\mathrm{A}$ \\
& $246.3 \mathrm{a}$ & $259.9 \mathrm{~b}$ & $256.0 \mathrm{~b}$ & $287.0 \mathrm{a}$ \\
TWC 352 & $\mathrm{A}$ & $\mathrm{A}$ & $\mathrm{C}$ & $\mathrm{B}$ \\
\hline
\end{tabular}

Table 6-b. Effect of interaction between $\mathbf{N}$ fertilizer levels and phosphorus fertilization regimes on ear grain weight $(\mathrm{g})$ in mono eared plants (combined data)

\begin{tabular}{|c|c|c|c|c|}
\hline \multirow[t]{2}{*}{ Phosphorus fertilization regimes } & \multicolumn{4}{|c|}{ N levels (kg N/fad.) } \\
\hline & 30 & 60 & 90 & 120 \\
\hline & $\mathrm{D}$ & $\mathrm{C}$ & $\mathrm{A}$ & $\mathrm{B}$ \\
\hline \multirow[t]{2}{*}{$30 \mathrm{~kg} \mathrm{P}_{2} \mathrm{O}_{5} / \mathrm{fad}$} & $223.1 \mathrm{c}$ & $241.9 \mathrm{~b}$ & $251.0 \mathrm{a}$ & $244.9 b$ \\
\hline & $\mathrm{A}$ & $\mathrm{C}$ & $\mathrm{D}$ & B \\
\hline \multirow[t]{2}{*}{ Phosphorien } & $240.5 \mathrm{a}$ & $233.0 \mathrm{c}$ & $228.1 \mathrm{c}$ & $238.2 \mathrm{c}$ \\
\hline & $\mathrm{D}$ & $\mathrm{B}$ & $\mathrm{C}$ & $\mathrm{A}$ \\
\hline $15 \mathrm{~kg} \mathrm{P}_{2} \mathrm{O}_{5} /$ fad., + phosphorien & 230.5 & $255.2 \mathrm{a}$ & $245.0 \mathrm{~b}$ & $264.5 \mathrm{a}$ \\
\hline
\end{tabular}

Table 6-c. Effect of interaction between maize hybrids and phosphorus fertilization regimes on ear grain weight (g) in mono eared plants (combined data)

\begin{tabular}{|c|c|c|c|}
\hline Maize hybrids (H) & $30 \mathrm{~kg} \mathrm{P}_{2} \mathrm{O}_{5} /$ fad., & Phosphorien & $15 \mathrm{~kg} \mathrm{P}_{2} \mathrm{O}_{5} /$ fad. + phosphorien \\
\hline & $\mathrm{B}$ & $\mathrm{C}$ & $\mathrm{A}$ \\
\hline \multirow[t]{2}{*}{ SC 168} & $254.3 b$ & $252.2 \mathrm{a}$ & $261.6 b$ \\
\hline & $\mathrm{B}$ & $\mathrm{C}$ & $\mathrm{A}$ \\
\hline \multirow[t]{2}{*}{ SC 167} & $259.4 \mathrm{a}$ & $249.7 b$ & $277.8 \mathrm{a}$ \\
\hline & A & $\mathrm{B}$ & $\mathrm{A}$ \\
\hline TWC 352 & $207.1 \mathrm{c}$ & $203.0 \mathrm{c}$ & $206.9 \mathrm{c}$ \\
\hline
\end{tabular}


Table 7. Grain yield (ardab/faddan) of maize as influenced by the different treatments during 2013 and 2014 seasons

\begin{tabular}{lccc}
\hline Main effects and interactions & $\mathbf{2 0 1 3}$ & $\mathbf{2 0 1 4}$ & Combined \\
\hline Maize hybrid (H) & $38.31 \mathrm{~b}$ & $40.54 \mathrm{~b}$ & $39.42 \mathrm{~b}$ \\
SC 168 & $39.45 \mathrm{a}$ & $41.26 \mathrm{a}$ & $40.36 \mathrm{a}$ \\
SC 167 & $31.64 \mathrm{c}$ & $32.63 \mathrm{c}$ & $32.13 \mathrm{c}$ \\
TWC 352 & $* *$ & $* *$ & $* *$ \\
F. test & & & \\
Nitrogen level (kg N/fad.) (N) & $34.22 \mathrm{~d}$ & $36.01 \mathrm{~d}$ & $35.12 \mathrm{~d}$ \\
$\mathbf{3 0}$ & $35.80 \mathrm{c}$ & $37.00 \mathrm{c}$ & $36.58 \mathrm{c}$ \\
$\mathbf{6 0}$ & $36.81 \mathrm{~b}$ & $38.40 \mathrm{~b}$ & $37.61 \mathrm{~b}$ \\
$\mathbf{9 0}$ & $39.02 \mathrm{a}$ & $40.81 \mathrm{a}$ & $39.91 \mathrm{a}$ \\
$\mathbf{1 2 0}$ & $* *$ & $* *$ & $* *$ \\
$\mathbf{F}$. test & & & \\
Phosphorus fertilization regimes (p) & $37.72 \mathrm{a}$ & $38.93 \mathrm{a}$ & $38.32 \mathrm{a}$ \\
$\mathbf{3 0}$ kg $\mathbf{P}_{\mathbf{2}} \mathbf{O}_{\mathbf{5}} /$ fad. & $37.54 \mathrm{a}$ & $39.10 \mathrm{a}$ & $38.32 \mathrm{a}$ \\
$\mathbf{P h o s p h o r i e n}$ & $34.13 \mathrm{~b}$ & $36.40 \mathrm{~b}$ & $35.26 \mathrm{~b}$ \\
$\mathbf{1 5 k g} \mathbf{P}_{\mathbf{2}} \mathbf{O}_{\mathbf{5}} /$ fad., + phosphorien & $* *$ & $* *$ & $* *$ \\
$\mathbf{F}$. test & & & $* *$ \\
$\mathbf{I n t e r a c t i o n s}$ & $* *$ & $* *$ & $* *$ \\
$\mathbf{H} \times \mathbf{N}$ & $* *$ & $* *$ & $* *$ \\
$\mathbf{H} \times \mathbf{P}$ & $* *$ & $* *$ & \\
$\mathbf{N} \times \mathbf{P}$ & & & \\
\hline
\end{tabular}

The significant positive impact of chemical phosphorus fertilizer on grain yield/fad., was reported by (Iqbal et al., 2003; Salih et al., 2007; Olusegun, 2015). Some authors reported that, application of biofertilizer of phosphorus increased growth and yield (Glaa, 1989; Sundara et al., 2002; Bolan and Duraisamy, 2003; Behbahani and Khayyam, 2004; Pradhan and Sukla, 2005; Xiao et al., 2011).

\section{Interaction effect}

Under the four $\mathrm{N}$ levels, SC 167 had the heaviest grain yield/fad., followed by SC 168 and TWC 352. Therefore, the highest grain yield/fad., of (44.26 ardab) was achieved by SC 167 hybrid when fertilized by $120 \mathrm{~kg} / \mathrm{fad}$. Otherwise, TWC 352 hybrid gave the lowest grain yield (29.24) when fertilized by $30 \mathrm{~kg}$ N/fad., (Table 7-a).

Under 30 and $60 \mathrm{~kg} \mathrm{~N} /$ fad., the highest grain yield recorded when maize plants were fertilized by $30 \mathrm{~kg} \mathrm{P} \mathrm{O}_{5} /$ fad., but recorded by 90 and 120 $\mathrm{kg} \mathrm{N} /$ fad., when fertilized by phosphorien only. When maize plants fertilized by $30 \mathrm{~kg} \mathrm{P}_{2} \mathrm{O}_{5} /$ fad., observed that $30 \mathrm{~kg} \mathrm{~N} /$ fad., was quiet enough to produce the heaviest grain yield, but when fertilized by phosphorien only they needed 120 $\mathrm{kg} \mathrm{N} /$ fad., and $60 \mathrm{~kg} \mathrm{~N} /$ fad., when fertilized by $15 \mathrm{~kg} \mathrm{P}_{2} \mathrm{O}_{5} /$ fad., + phosphorien, receptively. Then the higher grain yield (47.06) was recorded when maize plants, fertilized by each of phosphorien and $120 \mathrm{~kg} \mathrm{~N} / \mathrm{fad}$., where the lowest grain yield (32.98) was recorded when plants were fertilized by $30 \mathrm{~kg} \mathrm{~N} /$ fad., and $15 \mathrm{~kg}$ $\mathrm{P}_{2} \mathrm{O}_{5} /$ fad., + phosphorien (Table 7-b).

Under all phosphorus treatments, SC 167 recorded the highest grain yield, then the lowest grain yield was achieved by TWC 352 in this respect. Therefore, the heaviest grain yield (42.82) was obtained when SC 167 hybrid fertilized by phosphorien only.

Otherwise, the lowest grain yield (30.77) recorded by TWC 352 when fertilized by phosphorien only (Table 7-c). 
Table 7-a. Effect of interaction between $\mathbf{N}$ fertilizer levels and maize hybrids on grain yield (ardab/fad.) (combined data)

\begin{tabular}{lcccc}
\hline Maize hybrid (H) & \multicolumn{4}{c}{ N levels (kg N/fad.) } \\
\cline { 2 - 5 } & $\mathbf{3 0}$ & $\mathbf{6 0}$ & $\mathbf{9 0}$ & $\mathbf{1 2 0}$ \\
\hline \multirow{2}{*}{ SC 168 } & $\mathrm{D}$ & $\mathrm{C}$ & $\mathrm{B}$ & $\mathrm{A}$ \\
& $37.02 \mathrm{~b}$ & $38.07 \mathrm{~b}$ & $39.71 \mathrm{a}$ & $42.89 \mathrm{~b}$ \\
SC 167 & $\mathrm{B}$ & $\mathrm{C}$ & $\mathrm{B}$ & $\mathrm{A}$ \\
& $39.08 \mathrm{a}$ & $38.66 \mathrm{a}$ & $39.43 \mathrm{a}$ & $44.26 \mathrm{a}$ \\
TWC 352 & $\mathrm{D}$ & $\mathrm{B}$ & $\mathrm{A}$ & $\mathrm{C}$ \\
\hline
\end{tabular}

Table 7-b. Effect of interaction between $\mathbf{N}$ fertilizer levels and phosphorus fertilization regimes on grain yield (ardab/ fad.) (combined data)

\begin{tabular}{|c|c|c|c|c|}
\hline \multirow[t]{2}{*}{ Phosphorus fertilization regimes } & \multicolumn{4}{|c|}{ N levels (kg N/fad.) } \\
\hline & 30 & 60 & 90 & 120 \\
\hline & $\mathrm{A}$ & $\mathrm{A}$ & $\mathrm{B}$ & $\mathrm{C}$ \\
\hline \multirow[t]{2}{*}{$30 \mathrm{~kg} \mathrm{P}_{2} \mathrm{O}_{5} /$ fad. } & $39.61 \mathrm{a}$ & $39.24 \mathrm{a}$ & $38.31 b$ & $36.59 b$ \\
\hline & $\mathrm{C}$ & $\mathrm{C}$ & $\mathrm{B}$ & A \\
\hline \multirow[t]{2}{*}{ Phosphorien } & $33.21 \mathrm{~b}$ & $33.21 \mathrm{c}$ & $39.82 \mathrm{a}$ & $47.06 \mathrm{a}$ \\
\hline & $\mathrm{D}$ & $\mathrm{A}$ & $\mathrm{C}$ & $\mathrm{B}$ \\
\hline $15 \mathrm{~kg} \mathrm{P}_{2} \mathrm{O}_{5} /$ fad.+ phosphorien & $32.98 b$ & $37.29 \mathrm{~b}$ & $34.69 \mathrm{c}$ & $36.09 \mathrm{c}$ \\
\hline
\end{tabular}

Table 7-c. Effect of interaction between maize hybrids and phosphorus fertilization regimes on grain yield (ardab/fad.) (combined data)

\begin{tabular}{|c|c|c|c|}
\hline \multirow[b]{2}{*}{ Maize hybrids (H) } & \multicolumn{3}{|c|}{ Phosphorus fertilization regimes } \\
\hline & $30 \mathrm{~kg} \mathrm{P}_{2} \mathrm{O}_{5} /$ fad., & Phosphorien & $15 \mathrm{~kg} \mathrm{P}_{2} \mathrm{O}_{5}+$ phosphorien \\
\hline & $\mathrm{B}$ & A & $\mathrm{C}$ \\
\hline \multirow[t]{2}{*}{ SC 168} & $40.22 b$ & $41.39 b$ & $36.66 \mathrm{a}$ \\
\hline & $\mathrm{B}$ & A & $\mathrm{C}$ \\
\hline \multirow[t]{2}{*}{ SC 167} & $41.71 \mathrm{a}$ & $42.82 \mathrm{a}$ & $36.55 \mathrm{a}$ \\
\hline & A & $\mathrm{B}$ & A \\
\hline TWC 352 & $33.05 \mathrm{c}$ & $30.77 \mathrm{c}$ & $32.58 \mathrm{~b}$ \\
\hline
\end{tabular}




\section{REFERENCES}

Abd El-Maksoud, M.F. and A.A. Sarhan (2008). Response of some maize hybrids to bio and chemical nitrogen fertilization. Zagazig J. Agric. Res., 35 (3) : 497- 451.

Abdou, E.M., A.A. Ibrahim, S.A.I. Ghanem, O.A.A. Zeiton and A.E.A. Omar (2012). Effect of planting density and nitrogen fertilization on yield and its attributes of some yellow maize hybrids. Zagazig J. Agric. Res., 39 (6): 1033-1046.

Ahmad, M., N. Hussain, A. Jan, K. Ahmed and S. Hussain (2007). Response of some maize to phosphorus levels and plant density. Sarhad J. Agric., 23 (1): 69-74.

Alias, A., M. Usman, E. Ullah and E.A. Warraich (2003). Response of corn (Zea mays L.) cultivars to different levels of nitrogen applied in Swaziland. $7^{\text {th }}$ Estern and Southern Agric. Regional Maize Conf., 1115 Feb., 377-381.

Amer, S.S.A. (2004). Growth, green pods yield and seeds yield of common ben (Phaseouls vulgaris L.) as affected by active dry yeast, salicylic acid and their interaction. J. Agric. Sci. Mansoura. Univ., 29 (3): 1407-1422.

Attia, A.N.E., S.A. El-Mloursy, G.M.A. Mahgoub and M.M.B. Darwich (2009). Effect of ridge spacing and plant density for two maize hybrids. J. Agric. Sci. Mansoura Univ., 34 (7): 8073-8080.

Behbahani, M. and N.M. Khayyam (2004). Effect of phosphate solubilizing bacteria on yield of potato (Solanum tuberoum L.) in in vivo conditions. Seed and plant improvement institute. Iran, 316.

Beyranvand, H., A. Farnia, S.H. Nakhiavan and M. Shaban (2013). Response of yield and yield components of maize (Zea maiz L.) to different bio fertilizers. Int. J. Adv. Biol. and Biol. and Biomed. and Biomed. Res., 19: 1068-1077.

Bolan, N.S. and P. Duraisamy (2003). Role of inorganic and organic soil amendments on immobilization and phytoavailability of heavy metals: a review involving specific case studies. Aust. J. Soil Res., 41: 533-555.
Chabot, R., H. Antoun and M.P.P. Cescas (1993). Stimulation de la croissance du maiset de la laitue romaine par des microorganisms dissolvant le phosphore inorganique. Canadian J. Microbiol., 39: 941947.

Darwich, M.M.B. (2013). Effect of $\mathrm{N}$ rates, compost and humic acid treatments on growth and yield components of Maize. Ph. D. Thesis in Agron., Fac. Agric. Mansoura Univ., Egypt.

El-Kholy, A.S.M.M. (2015). Fertigated nitrogen level, organic manure, and plant population for drip irrigated maize in sandy soils. M.Sc. Thesis, Fac. Agric., Zagazig Univ., Egypt.

El-Moursy, R.S.A. (2013). Agromic studies on maize. Ph.D. Thesis in Agron., Fac. Agric., Mansoura Univ., Egypt.

El-Sobky, E.E.A.M. (2014). Effect of some agronomic practices on productivity of maize. Ph.D. Thesis, Fac. Agric., Zagazig Univ., Egypt.

Freed, R.S.P., S.P. Eisensmith, S. Goetez, D. Reicosky, V.W. Smail and P. Wolberg (1989). User's Guide to MSTAT-C A software program for the design, management and analysis of agronomic research experiments. Michigan State Univ., USA.

Glass, A.D.M. (1989). Plant Nutrition: An Introduction to Current Concepets, Jones and Bartlett publishers, Boston, MA, USA, 234.

Golezani, K.G. and Z. Tajbakhsh (2012). Relationship of plant biomass and grain filling with grain yield of maize cultivars. Int. J. Agric. and Crop Sci., 4 (20): 1536-1539.

Gomez, K.H. and A.A. Gomez (1984). Statistical Procedures for Agriculture Research. John Willy and Sons, Inc., New York.

Hejazi, L. and A. Soleymain (2014). Effect of different amounts of nitrogen fertilizer on grain yield of forage corn cultivars in Isfahan. Int. J. Adv. Biol. and Biom. Res., 2 (3): 608614.

Hussain, N., A.Z. Khan, H. Akbar, N.G. Bangash, Z.H. Khan and M. Idrees (2007). Response of maize varieties to phosphorus and potassium levels. Sarhad J. Agric., 23 (4): 881-887. 
Ikramullah, M.I., A.A. Khakwani, M. Sadiq, I. Awan, M. Munir and A. Ghazanfarullah (2015). Effect of nitrogen fertilization rates on growth, quality and economic return of fodder maize (Zea mays L.). Sarhad J. Agric., 31 (1) : 22-29.

Iqbal, R.M. and H.Q.I. Chauhan (2003). Effect of phosphorus levels on yield components, grain yield and harvest index of two maize varieries. Asian J. Plant Sci., 2: 800-803.

Iqball, Z., A. Latif, S. Ali and M.M. Iqbal (2003). Effect of fertigated phosphorus on $p$ use efficiency and yield of wheat and maize. J. Sci. Technol., 25 (6): 297-302.

Jackson, M.L. (1958). Soil Chemical Analysis. Prentice-Hall, Inc., England Cliffs, V.S. Library of Congress, USA.

Jat, B.L. and M.S. Shaktawat (2003). Effect of residual phosphorus, sulphur and biofertilizers on productivity, economics and nutrient conten of pearl millet (Pennisetum glaucum L.) in fenugreek (Trigonella foenumgraecum L.) pearl millet cropping sequence, Indian J. Agric. Sci., 73 (3): 134-137.

Kandil, E.E.E. (2013). Response of some maize hybrids (Zea mays L.) to different levels of nitrogenous fertilization, J. Appl. Sci., Res., 9 (3): 1902-1908.

Karimian, N. (2000). Consequences of excessive consumption of phosphate chemical fertilizers. Water and Soil Inst. Iran, 12.

Khan, A., F. Munsif, K. Akhtar, M.Z. Afridi, Z. Ahmad, S. Fahad, R. Ullah, F. Ahmed K. and M. Din (2014). Response of fodder maize to various levels of nitrogen and phosphorus. Ame. J. P Sci., 5 : 2323-2329.

Khan, M.A., M. Abid, N.A. Hussain and M.U. Masood (2005). Effect of phosphorus levels on growth and yield of maize (Zea mays L.) cultivars under saline conditions. Int. J. Agri. Biol., 7 (3): 511-514.

Lin, W., Y. Okon and R.W.F. Hardy (1983). Enhanced minral uptake by zea mays and Sorghum bicolor roots inoculated with Azospirillum brasilense. Appl. Environ. Microbiol., 45: 1775-1779.
Lindsay, W.L. and W.A. Norvell (1978). Development of DTPA soil test for micronutrients. Soil Sci. Soc. Ame. Proc., 42: 421-428.

Modhej, A., A. Kaihani and S. Lack (2014). Effect of nitrogen fertilizer on grain yield and nitrogen use efficiency in corn (Zea mays L.) hybrids under irrigated conditions proc. Nattl. Acad. Sci., India, Sect. B. Biol. Sci., 48 (3): 531-536.

Mukhtar, T., M. Arif, S. Hussain, M. Atif, S. Rehamn and K. Hussain (2012). Yield and yield components of maize hybrids as influenced by plant spacing. J. Agric. Res., 50 (1) $59: 69$.

Olusegun, O.S. (2015). Nitrogen (N) and phosphorus (P) fertilizer application on maize (Zea mays L.) growth and yield at Ado-Ekiti, South-west, Nigeria. Ame. J. Exp. Agric., 6 (1) : 22-29.

Omar, A.E.A. (2014). Effect of FYM and phosphorus fertilization on yield and components of maize. Asian J. of Crop Sci., 6 : 15-26.

Pradhan, N. and L.B. Sukla (2005). Solubilization of inorganic phosphate by fungi isolated from agriculture soil, Afr. J. Biotechnol., 5 : 850-854.

Radma, I.A.M. and Y.M.I. Dagash (2013). Effect of different nitrogen and weeding levels on yield of five maize cultivars under irrigation. Universal J. Agric. Res., 1 (4): 199 -225 .

Salih, M.A., E.A. Babiker, N.A. Ali and A.E.M Elamin (2007). Response of two maize (Zea mays L.) varieries to different sources and rates of phosphorus proc of the $43^{\text {rd }}$ meetings of the national crop husbandry committee ministry of science and technology, Agric. Res. Coporation, Wed Medani., 11-28.

Seadh, S.E., W.A.E. Abido and D.R. Abdulrahman (2015). The role of foliar application in reducing maize nitrogen requirements. J. Pl. Prod., Mansoura Univ., 5 (7): 1168-1180.

Sharifi, R.S., M.S.M. Sedghi and A.O. Gholipouri (2009). Effect of population density on yield and yield attributes of maize hybrids. Res. J. Bio. Sci., 4 (4): 375-379. 
Shevananda (2008). Influence of bio-fertilizers on the availability of nutrients $(\mathrm{N}, \mathrm{P}$ and $\mathrm{K})$ in soil in relation to growth and yirld of Stevia rebaudiana grown in south India. Int. J. Appl. Res. Nat. Prod., 1 (1): 20-24.

Sorkhi, F. and M. Fateh (2014). Effect of nitrogen fertilizer on yield components of maize. Int. J. Biosci., 5 (6): 16-20.

Steel, R.G.D. and J.H. Torrie (1980). Principles and Procedures of Statistics: A Biometrical Approach, $2^{\text {nd }}$ Ed. Mc Graw-Hill Book Co. New York, USA.

Sundara, B., V. Natarajan and K. Hari (2002). Influence of phosphorus solubilizing bacteria on the changes in soil available phosphorus and sugarcane yields, Field Crops Res., 77 : 40-43.
Waller, R.A. and D.B. Duncan (1969). A bayer rule for symmetric multiple comparisons problems. J. Ame. Statit. Assoc., 64: 1484-1503.

Xiao, C.Q., R.A. Chi, X.H. Li, M. Xia and Z.W. Xia (2011). Bio solubilization of rock phosphate by three stress- tolerant fungal strains, Chemical phosphorus fertilizer. Appl. Biochem. Biotech., 165: 719-727.

Yosefi, M.R. and S.R. Mousavi (2011). Effect of bio-phosphate and chemical phosphorus fertilizer accompanied with micronutrient foliar application on growth, yield and yield components of maize (single cross 704). Aust. J. Crop Sci. Ajcs, 5 (2) : 175-180.

Zafar, M., M.K. Abbasi and R.A. Zahid (2011). Effect of combining on growth, yield, energy content and phosphorus uptake in maize at Rawalakot Azad jammu and Kashmir. Arch. Appl .Sci. Res., 3 (2): 199-212.

\section{تأثيـر بعـض المعـاملات الزراعية على المحصول ومساهماته لبعض هجن الذرة الثــامية الصفـراء}

$$
\begin{aligned}
& \text { محمد على إبراهيم على شام ـ عمر الفاروق زيتون ـ عبدالستار عبدالقادر الخواجةـ صابر عبدالحميد موافى إفى }
\end{aligned}
$$

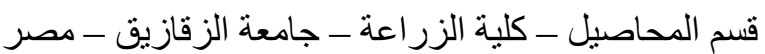

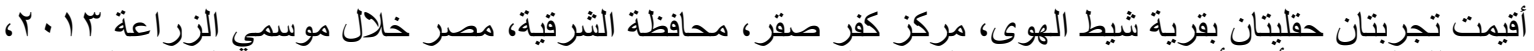

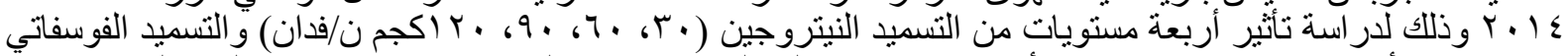

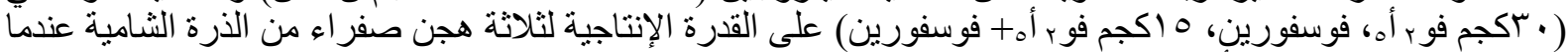

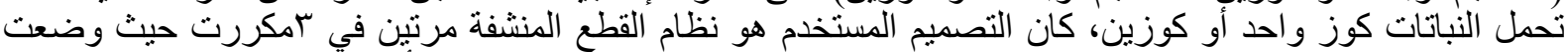

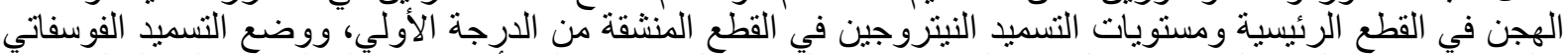

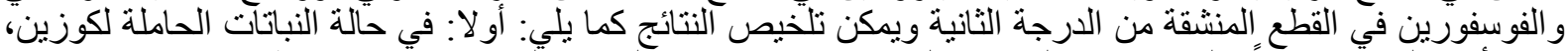

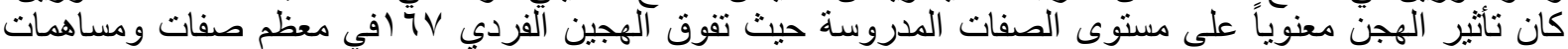

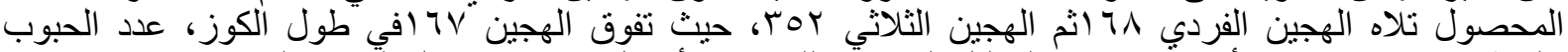

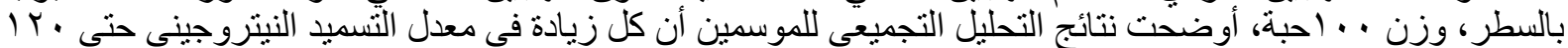

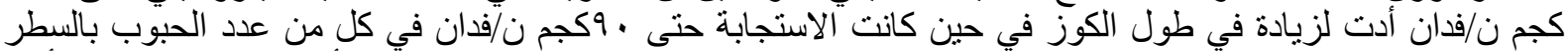

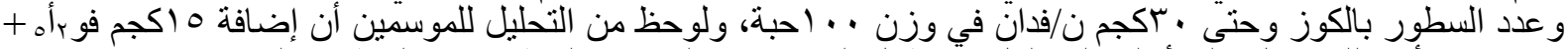

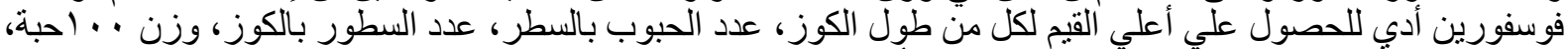

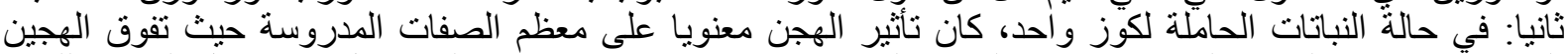

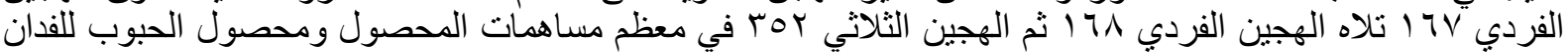

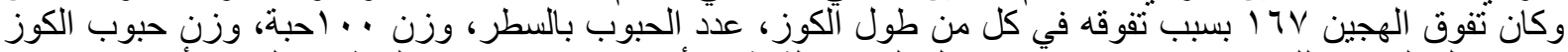

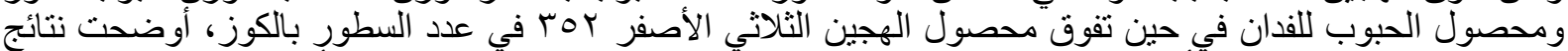

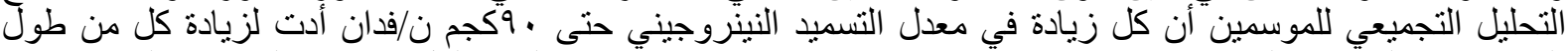

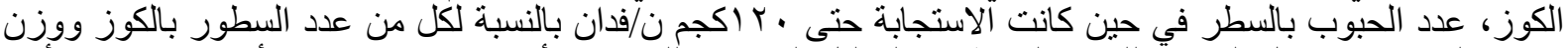

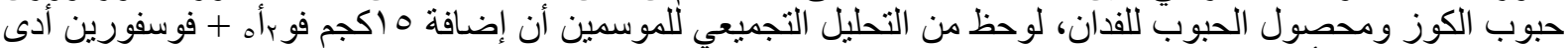

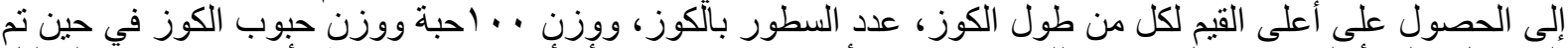

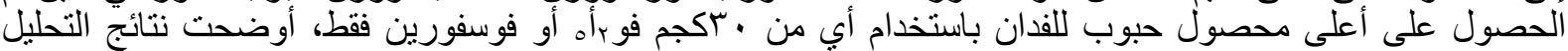

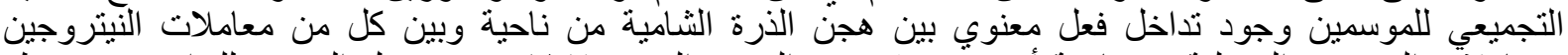

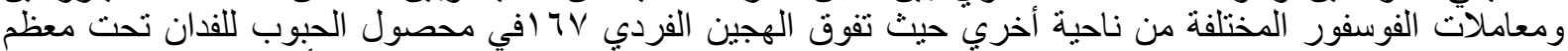

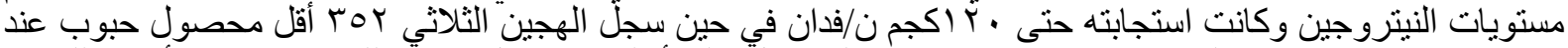

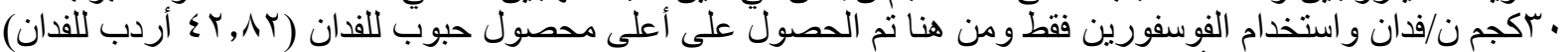

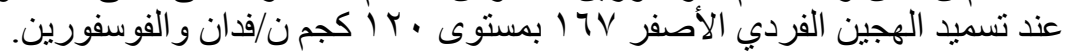

